TRANSACTIONS OF THE

AMERICAN MATHEMATICAL SOCIETY

Volume 360, Number 5, May 2008, Pages 2359-2392

S 0002-9947(07)04365-6

Article electronically published on November 20, 2007

\title{
SUPERCHARACTERS AND SUPERCLASSES FOR ALGEBRA GROUPS
}

\author{
PERSI DIACONIS AND I. M. ISAACS
}

\begin{abstract}
We study certain sums of irreducible characters and compatible unions of conjugacy classes in finite algebra groups. These groups generalize the unimodular upper triangular groups over a finite field, and the supercharacter theory we develop extends results of Carlos André and Ning Yan that were originally proved in the upper triangular case. This theory sometimes allows explicit computations in situations where it would be impractical to work with the full character table. We discuss connections with the Kirillov orbit method and with Gelfand pairs, and we give conditions for a supercharacter or a superclass to be an ordinary irreducible character or conjugacy class, respectively. We also show that products of supercharacters are positive integer combinations of supercharacters.
\end{abstract}

\section{INTRODUCTION}

Let $G$ be a finite group and as usual, write $\operatorname{Irr}(G)$ to denote the set of irreducible characters of $G$. Assume that $\operatorname{Irr}(G)$ is partitioned into a collection $\mathcal{X}$ of nonempty subsets, and suppose that for each member $X \in \mathcal{X}$, we have chosen a nonzero character $\chi_{X}$ whose irreducible constituents all lie in the set $X$. It is sometimes possible to find a compatible partition of the group $G$ into a collection $\mathcal{K}$ of nonempty subsets such that the characters $\chi_{X}$ are constant on each of the sets $K \in \mathcal{K}$, and where $|\mathcal{X}|=|\mathcal{K}|$. (We will also require a mild nondegeneracy condition: that $\{1\}$ is a member of $\mathcal{K}$.) In this situation, we refer to the functions $\chi_{X}$ as "supercharacters" of $G$ and the sets $K \in \mathcal{K}$ as "superclasses". Note that the supercharacters $\chi_{X}$ are automatically orthogonal with respect to the usual inner product for complex-valued functions defined on finite groups. (This is clear because the sets $X \subseteq \operatorname{Irr}(G)$ are disjoint and the irreducible characters of $G$ are orthogonal.)

For an arbitrary finite group $G$, there are two "trivial" supercharacter theories: in one, $\mathcal{X}$ consists just of singleton sets, and in the other $\mathcal{X}=\left\{\left\{1_{G}\right\}, \operatorname{Irr}(G)-\left\{1_{G}\right\}\right\}$, where $1_{G}$ is the principal character of $G$. The corresponding superclasses in the first case, of course, are just the conjugacy classes of $G$, and in the second case, they are the sets $\{1\}$ and $G-\{1\}$. (The supercharacters in the second example are $1_{G}$ and $\rho_{G}-1_{G}$, where $\rho_{G}$ is the regular character of $G$.)

Although for some groups these trivial examples are the only possibilities, there are many groups for which nontrivial supercharacter theories exist. For example,

Received by the editors December 30, 2005.

2000 Mathematics Subject Classification. Primary 20C15, 20D15.

(C) 2007 American Mathematical Society Reverts to public domain 28 years from publication 
suppose that $A$ is a group that acts via automorphisms on our given group $G$. Then, as is well known, $A$ permutes both the irreducible characters of $G$ and the conjugacy classes of $G$. By a lemma of R. Brauer, the permutation characters of $A$ corresponding to these two actions are identical, and hence there are equal numbers of $A$-orbits on $\operatorname{Irr}(G)$ and on the set of classes of $G$. (See Theorem 6.32 and Corollary 6.33 of [5].) It is easy to see that these orbit decompositions yield a supercharacter theory for $G$ : the members of $\mathcal{X}$ are the $A$-orbits on $\operatorname{Irr}(G)$, and the members of $\mathcal{K}$ are the unions of the $A$-orbits on the classes of $G$. It is clear that in this situation, the sum of the characters in an orbit $X \in \mathcal{X}$ is constant on each member of $\mathcal{K}$.

Another general way to construct a supercharacter theory for $G$ uses the action of a group $A$ of automorphisms of the cyclotomic field $\mathbb{Q}_{|G|}=\mathbb{Q}[\epsilon]$, where $\epsilon$ is a primitive $|G|$ th root of unity. Clearly, $A$ permutes $\operatorname{Irr}(G)$, and there is a compatible action on the classes of $G$, defined as follows. Given $\sigma \in A$, there is a unique positive integer $r<|G|$ such that $\sigma(\epsilon)=\epsilon^{r}$, and we let $\sigma$ carry the class of $g \in G$ to the class of $g^{r}$. Then Brauer's lemma shows that the numbers of $A$-orbits on $\operatorname{Irr}(G)$ and on the set of classes of $G$ are equal. In this case too, we take $\mathcal{X}$ to be the set of $A$-orbits on $\operatorname{Irr}(G)$, and again, $\mathcal{K}$ is the set of unions of the various $A$-orbits on conjugacy classes. As before, it is trivial to check that the sum of the characters in an orbit $X \in \mathcal{X}$ is constant on each member of $\mathcal{K}$, and so indeed, we have a supercharacter theory.

Sometimes, it may be possible to obtain useful information using supercharacters arising from a relatively coarse partition, and in such cases, the supercharacter table may be much easier to compute than the full character table. For some applications of Fourier analysis on finite groups, certain naturally occurring functions are constant on superclasses, and the new theory permits analysis even when the full character table is unavailable. For instance, a random-walk example can be found in [4].

Now consider $U_{n}(F)$, the group of $n \times n$ unimodular upper triangular matrices over a finite field $F$ of characteristic $p$. (By "unimodular", we mean that all diagonal entries are 1.) An explicit computation of the irreducible characters and the conjugacy classes of $U_{n}(F)$ is known to be a "wild" problem, but Carlos André [1] and Ning Yan [14] have developed an elegant (and applicable) supercharacter theory in this situation. André's original approach works only when $p \geq n$, although he extends this to the general case in a later paper [3]. Yan's construction, which is much more elementary, also works in general, and it yields the same supercharacter theory as André's. (André calls his supercharacters "basic characters" and Yan calls them "transition characters". We review some of their main results in Appendix A.)

In this paper, we generalize Yan's approach in order to extend the supercharacter theory of $U_{n}(F)$ to a much larger class of $p$-groups: algebra groups over a finite field $F$ of characteristic $p$. Let $J$ be a finite dimensional nilpotent associative algebra over $F$ (without unity, of course), and let $G$ be the set of formal objects of the form $1+x$, where $x \in J$. Then $G$ is easily seen to be a group with respect to the natural multiplication $(1+x)(1+y)=1+x+y+x y$. (In fact, $G$ is a subgroup of the group of units of the algebra $R=F \cdot 1+J$, in which $J$ is the Jacobson radical.) The group $G$ constructed in this way is the algebra group based on $J$. For example, if $J$ is the algebra of strictly upper triangular $n \times n$ matrices over $F$, then the 
corresponding algebra group is isomorphic to $U_{n}(F)$. In the case of $U_{n}(F)$, most of our results reduce to theorems of André and Yan.

Before we discuss supercharacters of algebra groups, let us return briefly to the general situation, and suppose that we have a supercharacter theory for some finite group $G$. In other words, we assume that there exist partitions $\mathcal{X}$ of $\operatorname{Irr}(G)$ and $\mathcal{K}$ of $G$ with $|\mathcal{X}|=|\mathcal{K}|$, and also that for each set $X \in \mathcal{X}$, we have a nonzero character $\chi_{X}$ with constituents in $X$ and such that $\chi_{X}$ is constant on each set $K$ in $\mathcal{K}$. In addition, we assume that $\{1\}$ is a member of $\mathcal{K}$. We shall see that each of the partitions $\mathcal{X}$ and $\mathcal{K}$ uniquely determines the other, and also determines the supercharacters $\chi_{X}$ up to a constant multiple. We shall also see that the superclasses of $G$ are always unions of conjugacy classes, and that working in the complex group algebra $\mathbb{C} G$, the product of two superclass sums is a nonnegative integer linear combination of superclass sums.

For algebra groups, we will resolve the constant-multiple ambiguity and define specific supercharacters in a natural way that generalizes the constructions of André and Yan. We shall see that the degrees of these supercharacters and the sizes of the superclasses are always powers of $q$, the order of the underlying field $F$. We will explore when it happens that a supercharacter is irreducible or a superclass is a single conjugacy class, and we will prove that the product of two supercharacters of an algebra group is always a nonnegative integer linear combination of supercharacters. Also, we will consider restriction and induction of supercharacters to and from algebra subgroups.

As we will explain, our construction of the supercharacters of an algebra group is a cruder version of the Kirillov orbit method, which attempts to construct the irreducible characters of such groups, but which does not always work. Kirillov's method for upper triangular groups is presented in [11]. (As we will mention later, however, a variation on his approach does work for $U_{n}(F)$ if $n \geq p$, or more generally for algebra groups with $J^{p}=0$, where $p$ is the characteristic of $F$.) Interestingly, it turns out that whenever one of our supercharacters happens to be irreducible, it is correctly described by Kirillov's original formula. We mention that André's construction of supercharacter theory is based on the Kirillov method, while Yan's (and ours) is independent of it.

We provide a brief summary of the work of André and Yan in Appendix A. In other appendices, we discuss connections with Gelfand pairs and spherical functions, we compute a few specific examples and we reformulate some of our results in the language of Fourier analysis.

\section{SUPERCHARACTERS IN GENERAL}

Let $G$ be a finite group and assume, as in Section 1 , that $\mathcal{X}$ is a partition of $\operatorname{Irr}(G)$. (Here, and throughout this paper, when we use the word "partition", we require that the parts are all nonempty.) For $X \in \mathcal{X}$, write $\sigma_{X}=\sum_{\psi \in X} \psi(1) \psi$, and note that $\sum_{X \in \mathcal{X}} \sigma_{X}=\rho_{G}$, the regular character of $G$. (Recall that $\rho(g)=0$ for $1 \neq g \in G$ and $\rho(1)=|G|$.) Also as in Section 1 , let $\mathcal{K}$ be a partition of $G$.

2.1. Lemma. For each set $X \in \mathcal{X}$, let $\chi_{X}$ be a nonzero character whose irreducible constituents lie in $X$. Assume that $|\mathcal{X}|=|\mathcal{K}|$ and that the functions $\chi_{X}$ are constant on the sets $K \in \mathcal{K}$. Then the following are equivalent.

(1) The set $\{1\}$ is a member of $\mathcal{K}$.

(2) Each character $\chi_{X}$ is a constant multiple of $\sigma_{X}$. 
(3) Each irreducible character $\psi$ of $G$ is a constituent of one of the characters $\chi_{X}$.

Proof. Because the irreducible characters of $G$ are linearly independent and the sets $X \in \mathcal{X}$ are disjoint, it follows that the characters $\chi_{X}$ are linearly independent. These characters lie in the space of complex-valued functions that are constant on the sets $K \in \mathcal{K}$, and since $|\mathcal{K}|=|\mathcal{X}|$, they form a basis for this space. Assuming (1) now, we see that the regular character $\rho_{G}$ is constant on all members of $\mathcal{K}$, and thus we can write

$$
\sum_{X \in \mathcal{X}} a_{X} \chi_{X}=\rho_{G}=\sum_{X \in \mathcal{X}} \sigma_{X}
$$

for appropriate complex scalars $a_{X}$. Since the sets $X \in \mathcal{X}$ are disjoint and the irreducible characters of $G$ are linearly independent, it follows that $a_{X} \chi_{X}=\sigma_{X}$ for all $X \in \mathcal{X}$, and this establishes (2).

It is a triviality that (2) implies (3), so we assume (3) now, and we work to establish (1). Let $K$ be the member of $\mathcal{K}$ that contains 1 , and let $g \in K$. Since each of the characters $\chi_{X}$ is constant on $K$, we have $\chi_{X}(g)=\chi_{X}(1)$, and thus $g$ is in the kernel of every irreducible constituent of $\chi_{X}$. By (3), however, every irreducible character of $G$ is a constituent of one of the characters $\chi_{X}$, and thus $g$ is in the kernel of all members of $\operatorname{Irr}(G)$. It follows that $g=1$, and the proof is complete.

It seems reasonable to require that every irreducible character of $G$ should appear as a constituent of some supercharacter, or equivalently, that $\{1\}$ should be a superclass, and so we will say that the partitions $\mathcal{X}$ of $\operatorname{Irr}(G)$ and $\mathcal{K}$ of $G$, together with the choices of characters $\chi_{X}$ as above form a supercharacter theory for $G$ provided that the following hold:

(a) $|\mathcal{X}|=|\mathcal{K}|$,

(b) the characters $\chi_{X}$ are constant on the members of $\mathcal{K}$ and

(c) the set $\{1\}$ is a member of $\mathcal{K}$.

As we have indicated, we refer to the characters $\chi_{X}$ as the supercharacters and to the members of $\mathcal{K}$ as superclasses. In the following sections, however, we will define a particular supercharacter theory for algebra groups, and we will reserve the terms "supercharacter" and "superclass" for the specific objects associated with that theory.

By Lemma 2.1, we see that if we have a supercharacter theory for a group $G$, it is no loss to assume that our supercharacters are the characters $\sigma_{X}$. (We will not make that assumption for algebra groups, however.) Given an arbitrary partition $\mathcal{X}$ of $\operatorname{Irr}(G)$, therefore, we can attempt to construct a corresponding supercharacter theory by considering partitions $\mathcal{K}$ of $G$ such that the characters $\sigma_{X}$ for $X \in \mathcal{X}$ are constant on the members of $\mathcal{K}$. (We refer to such a partition of $G$ as being compatible with $\mathcal{X}$.) Partitions compatible with $\mathcal{X}$ always exist, of course, since we could take the members of $\mathcal{K}$ to be the conjugacy classes of $G$. Indeed, given $\mathcal{X}$, it is easy to see that there is a unique coarsest partition $\mathcal{K}$ of $G$ compatible with $\mathcal{X}$. As we shall show, every partition $\mathcal{K}$ compatible with $\mathcal{X}$ satisfies $|\mathcal{K}| \geq|\mathcal{X}|$, but equality usually fails even for the coarsest possible partition $\mathcal{K}$, which has the fewest possible parts. In general, therefore, we cannot expect to construct a supercharacter theory for $G$ by starting with an arbitrary partition $\mathcal{X}$ of $\operatorname{Irr}(G)$. 
As we mentioned in the Introduction, every finite group has trivial supercharacter theories, and it is not hard to see that for some finite groups (for example the nonabelian group of order 6) the two trivial supercharacter theories are the only ones. The smallest group for which there exists a nontrivial supercharacter theory is the cyclic group of order 4 . For that group, we can take $|\mathcal{K}|=3=|\mathcal{X}|$, where the set consisting of the two elements of order 4 is one of the members of $\mathcal{K}$ and the set consisting of the two faithful linear characters is one of the members of $\mathcal{X}$.

We introduce some notation for the next theorem. If $K$ is an arbitrary subset of a group $G$, we write $\widehat{K}$ to denote the sum of the elements of $K$ in the complex group algebra $\mathbb{C} G$. Also, we recall that if $\psi \in \operatorname{Irr}(G)$, then there is a central idempotent $e_{\psi} \in \mathbb{C} G$ corresponding to $\psi$, and the coefficient of a group element $g$ in $e_{\psi}$ is $(1 /|G|) \chi(1) \overline{\chi(g)}$. If $X$ is a subset of $\operatorname{Irr}(G)$, we write $f_{X}$ to denote the sum of the idempotents $e_{\psi}$ for $\psi \in X$. Since the idempotents $e_{\psi}$ are orthogonal, we see that $f_{X}$ is idempotent. Also, idempotents of the form $f_{X}$ are orthogonal if the corresponding sets $X$ are disjoint.

2.2. Theorem. Let $\mathcal{X}$ and $\mathcal{K}$ be partitions of $\operatorname{Irr}(G)$ and of $G$, respectively, and assume that the characters $\sigma_{X}$ for $X \in \mathcal{X}$ are constant on the sets $K \in \mathcal{K}$. Then $|\mathcal{X}| \leq|\mathcal{K}|$. If $|\mathcal{X}|=|\mathcal{K}|$, then the following hold.

(a) The characters $\sigma_{X}$ for $X \in \mathcal{X}$ span the space of all complex-valued functions on $G$ that are constant on the members of $\mathcal{K}$.

(b) The linear span in $\mathbb{C} G$ of the elements $\widehat{K}$ for $K \in \mathcal{K}$ is a subalgebra of the center $\mathbf{Z}(\mathbb{C} G)$. Also, the idempotents $f_{X}$ for $X \in \mathcal{X}$ form a basis for this subalgebra.

(c) The partitions $\mathcal{X}$ and $\mathcal{K}$ uniquely determine each other, and in fact, $\mathcal{K}$ is the unique coarsest partition of $G$ that is compatible with $\mathcal{X}$. In particular, the members of $\mathcal{K}$ are unions of conjugacy classes.

(d) Some member of $\mathcal{K}$ consists of just the identity of $G$ and some member of $\mathcal{X}$ consists of just the principal character of $G$.

(e) Each automorphism of the field $\mathbb{C}$ induces a permutation on the set $\mathcal{X}$.

(f) If $r$ is an integer coprime to $|G|$, then the map $g \mapsto g^{r}$ induces a permutation on the set $\mathcal{K}$.

Proof. The characters $\sigma_{X}$ for $X \in \mathcal{X}$ form a linearly independent subset of the vector space $V$ of all complex valued functions on $G$ that are constant on members of $K$. Since $\operatorname{dim}(V)=|\mathcal{K}|$, it follows that $|\mathcal{X}| \leq|\mathcal{K}|$, as claimed. Assuming now that equality holds; we see that the $\sigma_{X}$ form a basis for $V$, and this proves (a).

Since the coefficient of the group element $g$ in the idempotent $e_{\psi}$ is $(1 /|G|) \psi(1) \overline{\psi(g)}$, we see that the coefficient of $g$ in $f_{X}$ is $(1 /|G|) \overline{\sigma_{X}(g)}$. Because $\sigma_{X}$ is constant on each of the sets $K \in \mathcal{K}$, it follows that $f_{X}$ is a linear combination of the elements $\widehat{K}$ of $\mathbb{C} G$, and thus $f_{X}$ lies in the linear span $A$ of these elements.

The $f_{X}$ are orthogonal idempotents, and hence they are linearly independent. The number of $f_{X}$ is $|\mathcal{X}|=|\mathcal{K}| \geq \operatorname{dim}(A)$, and it follows that the $f_{X}$ form a basis for $A$, as claimed. Since the idempotents $f_{X}$ are central, we have $A \subseteq \mathbf{Z}(\mathbb{C} G)$. Again using the fact that the $f_{X}$ are orthogonal idempotents, it follows that their linear span $A$ is a subalgebra, and this completes the proof of (b).

To see that $\mathcal{X}$ uniquely determines $\mathcal{K}$, let $\mathcal{K}_{0}$ be the unique coarsest partition of $G$ compatible with $\mathcal{X}$. In other words, the members of $\mathcal{K}_{0}$ are the equivalence classes under the relation on $G$ defined by $u \sim v$ if and only if $\sigma_{X}(u)=\sigma_{X}(v)$ for 
all $X \in \mathcal{X}$. (In particular, if $u$ and $v$ are conjugate in $G$, then $u \sim v$, and thus the members of $\mathcal{K}_{0}$ are unions of conjugacy classes.) Since each member of $\mathcal{K}$ is contained in some member of $\mathcal{K}_{0}$, we have $\left|\mathcal{K}_{0}\right| \leq|\mathcal{K}|=|\mathcal{X}|$. But $\left|\mathcal{K}_{0}\right| \geq|\mathcal{X}|$ by the first assertion of the theorem, and thus $\left|\mathcal{K}_{0}\right|=|\mathcal{X}|=|\mathcal{K}|$, and it follows that $\mathcal{K}=\mathcal{K}_{0}$. Thus $\mathcal{X}$ determines $\mathcal{K}$ and the members of $\mathcal{K}$ are unions of classes, as claimed. To see that $\mathcal{K}$ determines $\mathcal{X}$, observe that $\mathcal{K}$ determines the subalgebra $A$ and that the $f_{X}$ are the unique primitive idempotents in $A$. Since $X$ is exactly the set of irreducible characters $\psi$ of $G$ such that $f_{X} e_{\psi} \neq 0$, it follows that $\mathcal{K}$ uniquely determines the partition $\mathcal{X}$ of $\operatorname{Irr}(G)$, as required, and this completes the proof of (c).

The fact that the set $\{1\}$ lies in $\mathcal{K}$ follows by Lemma 2.1. Now let $Y \in \mathcal{X}$ contain the principal character $1_{G}$. If $Y>\left\{1_{G}\right\}$, we can construct a new partition $\mathcal{X}_{0}$ of $\operatorname{Irr}(G)$ by splitting $Y$ into the two sets $\left\{1_{G}\right\}$ and $Y-\left\{1_{G}\right\}$ and leaving all other members of $\mathcal{X}$ unchanged. Since $1_{G}$ is constant on $G$, it follows that all characters $\sigma_{X}$ for $X \in \mathcal{X}_{0}$ are constant on the members of $\mathcal{X}_{0}$, and thus $|\mathcal{X}|=|\mathcal{K}| \geq\left|\mathcal{X}_{0}\right|=$ $|\mathcal{X}|+1$, where the inequality follows from the first assertion of the theorem. This contradiction shows that $Y=\left\{1_{G}\right\}$, and the proof of (d) is complete.

Now suppose that $\tau$ is an automorphism of the complex numbers. Then $\tau$ permutes $\operatorname{Irr}(G)$, and so we obtain a new partition $\mathcal{Y}$ of $\operatorname{Irr}(G)$ by applying $\tau$ to each member of $\mathcal{X}$. If $Y \in \mathcal{Y}$, then $Y=X^{\tau}$ for some set $X \in \mathcal{X}$, and we see that $\sigma_{Y}=\left(\sigma_{X}\right)^{\tau}$ is constant on the sets $K \in \mathcal{K}$. Since $|\mathcal{Y}|=|\mathcal{X}|=|\mathcal{K}|$, we can conclude from (c) that $\mathcal{Y}=\mathcal{X}$, and this proves $(\mathrm{e})$.

Finally, if $r$ is an integer coprime to $|G|$, then the map $g \mapsto g^{r}$ defines a permutation of $G$, and so if we apply this map to the members of $\mathcal{K}$, we get a new partition $\mathcal{L}$ of $G$. Also, there exists a field automorphism $\tau$ such that $\psi\left(g^{r}\right)=\psi(g)^{\tau}$ for all characters $\psi \in \operatorname{Irr}(G)$ and all elements $g \in G$. For $K \in \mathcal{K}$ and $X \in \mathcal{X}$, therefore, it follows that $\sigma_{X}\left(g^{r}\right)=\sigma_{X}(g)^{\tau}$ is constant as $g$ runs over $K$. The characters $\sigma_{X}$ are thus constant on the sets forming the partition $\mathcal{L}$ of $G$. Since $|\mathcal{L}|=|\mathcal{K}|=|\mathcal{X}|$, we conclude from (c) that $\mathcal{L}=\mathcal{K}$, proving (f).

Note that it follows from parts (e) and (f) of the previous theorem that the complex conjugate of a supercharacter $\sigma_{X}$ is again a supercharacter and that the set of inverses of the members of a superclass is again a superclass.

2.3. Corollary. Suppose that $K$ and $L$ are superclasses in some supercharacter theory for a group $G$. Then $\widehat{K} \widehat{L}$ is a nonnegative integer linear combination of superclass sums in the group algebra $\mathbb{C} G$.

Proof. By Theorem 2.2(b), we know that $\widehat{K} \widehat{L}$ is a $\mathbb{C}$-linear combination of superclass sums. If $M$ is an arbitrary superclass, we see that the coefficient of $\widehat{M}$ in $\widehat{K} \widehat{L}$ is exactly the coefficient of the group element $m$ in this product, where $m$ is any fixed element of $M$. It is clear, however, that this coefficient is exactly equal to the number of ordered pairs $(k, l)$, where $k \in K, l \in L$ and $k l=m$. This, of course, is a nonnegative integer, as required.

Finally, we present an analog of a standard integrality result of ordinary character theory,

2.4. Theorem. Let $\chi$ be a supercharacter and $K$ a superclass belonging to some supercharacter theory for a group $G$, and let $k \in K$. Then $\chi(k)|K| / \chi(1)$ is an algebraic integer. 
Proof. Let $\mathcal{X}$ and $\mathcal{K}$ be as before, where $K \in K$ and $\chi=\chi_{Y}$ for some member $Y \in$ $\mathcal{X}$. Let $A$ be the linear span of the superclass sums and recall from Theorem 2.2(b) that the idempotents $f_{X}$ for $X \in X$ form a basis for $A$. Let $\omega: A \rightarrow \mathbb{C}$ be the function that picks out for each element $a \in A$, the coefficient of $f_{Y}$ when $a$ is expressed as a linear combination of the $f_{X}$. Because the basis $\left\{f_{X} \mid X \in\right.$ $\mathcal{X}$ \} consists of idempotents, it follows that $\omega$ is a $\mathbb{C}$-algebra homomorphism. By Corollary 2.3, therefore, the $\mathbb{Z}$-submodule of $\mathbb{C}$ generated by the complex numbers $\omega(\widehat{L})$ for $L \in \mathcal{K}$ is closed under multiplication. We conclude that $\omega(\widehat{K})$ is an algebraic integer.

Extending the character $\chi$ by linearity to all of $\mathbb{C} G$, we have $\chi\left(f_{X}\right)=0$ if $X \neq Y$, and thus $\chi\left(f_{Y}\right)=\chi(1)$. It follows that $\chi(\widehat{K})=\omega(\widehat{K}) \chi(1)$. But $\chi$ is constant on $K$, and thus $\chi(\widehat{K})=\chi(k)|K|$. Thus $\chi(k)|K| / \chi(1)=\omega(\widehat{K})$ is an algebraic integer.

\section{Algebra groups And orbits}

Let $F$ be a finite field of order $q$ and characteristic $p$, and let $J$ be a finite dimensional nilpotent associative $F$-algebra. Form the algebra group $G=1+J$ (as explained in the Introduction) and observe that $G$ is a $p$-group with $|G|=|J|=q^{d}$, where $d=\operatorname{dim}_{F}(J)$.

If we view $G$ as a subgroup of the group of units of the algebra $F \cdot 1+J$, we see that right multiplication defines a right action of $G$ on $J$ and that left multiplication defines a left action of $G$ on $J$. These actions are compatible in the sense that $(h x) g=h(x g)$ for all $g, h \in G$ and $x \in J$. They decompose $J$ into right orbits $x G$ and left orbits $G x$ for $x \in G$. We will also consider the two-sided orbits $G x G$ for $x \in J$. Finally, we mention the conjugation action of $G$ on $J$ and its corresponding conjugation orbits. Note that each two-sided orbit is a union of right orbits, a union of left orbits and a union of conjugation orbits.

It should be clear that the conjugacy classes of the algebra group $G=1+J$ are exactly the subsets of the form $1+\mathcal{O}$, where $\mathcal{O}$ is a conjugation orbit of $G$ on $J$. We define the superclasses of $G$ to be the subsets of the form $1+G x G$ for elements $x \in J$, and we observe that since the two-sided orbit $G x G$ is a union of conjugation orbits, each superclass is a union of conjugacy classes. (Eventually, we will define the supercharacters of an algebra group, and we will see that these definitions are compatible with the general definitions of the previous section.)

Of course, the size of the conjugacy class of an element $g \in G$ is equal to $|G| /\left|\mathbf{C}_{G}(g)\right|$. Also, $\mathbf{C}_{G}(g)=1+\mathbf{C}_{J}(g)$, and we see that $\mathbf{C}_{J}(g)$ is a subspace (and in fact, is a subalgebra) of $J$. Thus $\left|\mathbf{C}_{J}(g)\right|$ is a power of $q$, and hence all class sizes of $G$ are powers of $q$. Note also that if $g=1+x$ with $x \in J$, then $\mathbf{C}_{J}(g)=\mathbf{C}_{J}(x)$.

To prove that the superclass sizes of $G$ are also powers of $q$, it is convenient to have the following lemma, which appears in Yan's paper [14].

3.1. Lemma. Let $G$ be a finite group and suppose that $G$ acts compatibly on the left and right of some set $\Omega$. Let $\alpha \in \Omega$ with two-sided orbit $G \alpha G$. Then

$$
|G \alpha G|=\frac{|\alpha G||G \alpha|}{|\alpha G \cap G \alpha|} .
$$

Proof. The two-sided orbit $G \alpha G$ is a union of left orbits that are transitively permuted by the right action of $G$. Because they are transitively permuted, these left orbits all have the same size, namely $|G \alpha|$, and thus $|G \alpha G|=n|G \alpha|$, where $n$ is 
the number of left orbits in $G \alpha G$. The right action of $G$ is also transitive on the set of intersections of $\alpha G$ with the $n$ right translates of $G \alpha$, and these $n$ sets of cardinality $|\alpha G \cap G \alpha|$ partition $|\alpha G|$. It follows that $|\alpha G|=n|\alpha G \cap G \alpha|$ and we have $n=|\alpha G| /|\alpha G \cap G \alpha|$. The result now follows.

3.2. Corollary. Let $G=1+J$ be an algebra group, where the underlying field $F$ has order $q$. If $x \in J$, then

$$
|G x G|=\frac{|x J||J x|}{|x J \cap J x|}
$$

and in particular, the superclasses of $G$ all have q-power size.

Proof. Let $x \in J$. Then $x G=x+x J$ is an additive coset of the $F$-subspace $x J$ in $J$, and hence $|x G|=|x J|$ is a power of $q$. Similarly, $G x=x+J x$, and so $|G x|=|J x|$ is a power of $q$. Also, $x G \cap G x=(x+x J) \cap(x+J x)=x+(x J \cap J x)$ is a coset of the $F$-subspace $x J \cap J x$, and so $|x G \cap G x|=|x J \cap J x|$ is also a power of $q$. The result now follows by Lemma 3.1.

Next, we present a condition sufficient to guarantee that a superclass of an algebra group $G=1+J$ is just an ordinary conjugacy class. To do this, we introduce a bit of notation. Given $x \in J$, write $L_{x}=\{y \in J \mid y x=0\}$ and $R_{x}=\{y \in J \mid x y=0\}$. These are respectively the left annihilator and the right annihilator of $x$ in $J$.

3.3. Theorem. Let $G=1+J$ be an algebra group, and let $x \in J$. Suppose that $L_{x}+R_{x}=J$, where $L_{x}$ and $R_{x}$ are the left and right annihilators of $x$ in $J$, as above. Then the superclass of $1+x$ is the conjugacy class of $1+x$.

Proof. We must show that $G x G$ is the conjugation orbit $\mathcal{O}$ of $x$. Since $\mathcal{O} \subseteq G x G$, it suffices to show that $|\mathcal{O}| \geq|G x G|$, or equivalently, that

$$
\frac{|J|}{|C|} \geq \frac{|x J||J x|}{|x J \cap J x|}
$$

where $C=\mathbf{C}_{J}(x)$. Since $|x J|=|J| /\left|R_{x}\right|$ and $|J x|=|J| /\left|L_{x}\right|$, our goal is to show that $|C||J| \leq\left|L_{x}\right|\left|R_{x}\right||x J \cap J x|$.

We are assuming that $J=R_{x}+L_{x}$, and so $|J|=\left|R_{x}\right|\left|L_{x}\right| /\left|R_{x} \cap L_{x}\right|$. It thus suffices to show that $|C| /\left|R_{x} \cap L_{x}\right| \leq|x J \cap J x|$. Now observe that $R_{x} \cap L_{x}=C \cap L_{x}$, and thus $|C| /\left|R_{x} \cap L_{x}\right|=|C x|$. But clearly, $C x \subseteq x J \cap J x$, and the result follows.

The converse of Theorem 3.3 is false; the superclass of $1+x$ can be a conjugacy class even if $L_{x}+R_{x} \neq J$. Consider, for example, the situation where $J$ has basis $\{x, y, z\}$ over a field $F$ of order $q$, and assume that all products of these basis vectors in $J$ are 0 except that $x y=z$ and $y x=\alpha z$, where $\alpha \in F$ is some fixed scalar. If $\alpha \neq 1$, then $x$ is not central in $J$, and thus $C=\mathbf{C}_{J}(x)=F z+F x$ has dimension 2. In this case, the conjugation orbit $\mathcal{O}$ of $x$ has size $q$. If $\alpha \neq 0$, we see that $x J=F z=J x$, and thus $|G x G|=q$ by Corollary 3.2. It follows that $\mathcal{O}=G x G$ if $\alpha \notin\{0,1\}$, and so the superclass of $1+x$ is the conjugacy class of this element. In this case, $R_{x}<J$ and $L_{x}<J$, and it follows that $R_{x}=F x+F z=L_{x}$, and thus $R_{x}+L_{x}<J$, as claimed.

The algebra $J=J_{\alpha}$ actually exists for every choice of $\alpha \in F$. It can be realized, for example, as a subalgebra of the algebra of strictly upper triangular $4 \times 4$ matrices over $F$. To see this, write $e_{i, j}$ to denote the matrix units, as usual, and take $x=e_{1,2}+\alpha e_{3,4}, y=e_{1,3}+e_{2,4}$ and $z=e_{1,4}$. Of course, these matrices are linearly 
independent, and it is routine to check that they satisfy the multiplication rules stated in the previous paragraph. In particular, they span a subalgebra of the upper triangular matrices.

We can use these algebras $J_{\alpha}$ to illustrate another interesting point. Suppose that $|F|=p$ is a prime exceeding 2 . Then for all $\alpha \neq 1$, the groups $G=1+J$ are nonabelian and have order $p^{3}$ and exponent $p$, and so all of these groups are isomorphic. It is not hard to show, however, that the algebras $J_{\alpha}$ tend to be nonisomorphic as $\alpha$ varies. In fact, $J_{\alpha} \cong J_{\beta}$ if and only if $\beta=\alpha$ or $\alpha \beta=1$. In general, therefore, the algebra $J$ contains more information than does the algebra group $G=1+J$. Although we omit the proof of our isomorphism assertion, we mention that it is easy to see that $J_{0}$ is not isomorphic to $J_{\alpha}$ for $\alpha \neq 0$. This is because the right annihilator of the whole algebra $J_{\alpha}$ has dimension 2 if $\alpha=0$ and has dimension 1 , otherwise.

We close this section with some examples. First, we give an application of Theorem 3.3 in the case where $G=U_{n}(F)$, the group of unimodular upper triangular $n \times n$ matrices over $F$. Here, and whenever we consider this group, we view it as the algebra group $1+J$, where $J$ is the algebra of strictly upper triangular $n \times n$ matrices.

3.4. Corollary. Let $G$ be the unimodular upper triangular matrix group $U_{n}(F)$. Suppose that $g \in G$ has exactly one nonzero above-diagonal entry. Then the superclass of $g$ in $G$ is just the ordinary conjugacy class of $g$.

Actually, Corollary 3.4 is valid in a more general setting, which we now describe. We call a subgroup $G$ of $U_{n}(F)$ a pattern subgroup if it consists of all of the matrices in $U_{n}(F)$ for which the $(i, j)$ entry is zero for positions $(i, j)$ (with $i<j$ ) in some specified set of "forbidden" positions. For example, it is easy to check that we get a group of order $|F|^{2 n-3}$ if we require that the $(i, j)$-entry is zero whenever $1<i<j<n$. (If $|F|=p$ is prime, this pattern group is an extraspecial $p$-group.) Of course, the full unimodular upper triangular group is a pattern group with empty set of forbidden positions.

An alternative way to think about a pattern group is to consider a set $\mathcal{P}$ of "allowed" positions $(i, j)$ with $i<j$. Suppose that the set $\mathcal{P}$ has the property that if $(i, j)$ and $(j, k)$ are in $\mathcal{P}$, then $(i, k) \in \mathcal{P}$. In this case, the linear span $J$ of the matrix units corresponding to positions in $\mathcal{P}$ is a subalgebra of the algebra of strictly upper triangular matrices, and the corresponding algebra group $1+J$ is exactly the pattern group with forbidden positions $(i, j)$, where $(i, j) \notin \mathcal{P}$ and $i<j$. The following includes Corollary 3.4.

3.5. Corollary. Let $G$ be a pattern group, viewed as an algebra group, as above, and suppose that $g \in G$ has exactly one nonzero above-diagonal entry. Then the superclass of $g$ is the class of $g$ in $G$.

Proof. Write $g=1+a e_{i, j}$ with $a \in F$ and $i<j$. Now consider an arbitrary matrix unit $e_{u, v} \in J$. Since $u<v$, we cannot have both $u=j$ and $v=i$, and thus either $x e_{u, v}=0$ or $e_{u, v} x=0$. In other words, either $e_{u, v} \in R_{x}$ or $e_{u, v} \in L_{x}$. The various matrix units $e_{u, v}$ in $J$ form a basis for $J$, however, and so $R_{x}+L_{x}=J$. The result now follows by Theorem 3.3 .

Finally, we give an example where a superclass consists of more than one class. Let $G=U_{4}(F)$ and let $J$ be as usual. Consider $x=e_{1,2}+e_{3,4} \in J$. The matrix 
units $e_{1,2}, e_{1,4}$ and $e_{3,4}$ annihilate $x$ on both sides, and thus they centralize $x$. Also, $\left(e_{1,3}+e_{2,4}\right) x=e_{1,4}=x\left(e_{1,3}+e_{2,4}\right)$, and so the space $C=\mathbf{C}_{J}(x)$ has dimension at least 4. As we have seen, the size of the class of $1+x$ in $G$ is $|J| /|C|$, and since $\operatorname{dim}(J)=6$, it follows that the class of $1+x$ has size at most $q^{2}$, where $|F|=q$. (Actually, the size of this class is exactly $q^{2}$.) The size of the superclass of $1+x$ is $|G x G|=|x J||J x| /|x J \cap J x|$ by Corollary 3.2. It is easy to see that $x J=F \cdot e_{1,3}+F \cdot e_{1,4}$ and $J x=F \cdot e_{1,4}+F \cdot e_{2,4}$. Thus $|x J|=q^{2}=|J x|$ and $|x J \cap J x|=q$. The superclass size for $1+x$, therefore, is $q^{3}$, and this exceeds the class size.

\section{LinEAR FUnCtionals}

As before, let $J$ be a finite dimensional nilpotent associative algebra over a field $F$ of finite order $q$. In this section, we consider the dual space $J^{*}$ of $J$, which, we recall, is the space of $F$-linear functionals $\lambda: J \rightarrow F$.

For each of the actions of $G=1+J$ on $J$ that we discussed in the previous section, there is a corresponding action of $G$ on $J^{*}$. Given $\lambda \in J^{*}$ and $g \in G$, we define linear functionals $\lambda g$ and $g \lambda$ by the formulas $(\lambda g)(x)=\lambda\left(x g^{-1}\right)$ and $(g \lambda)(x)=\lambda\left(g^{-1} x\right)$ for $x \in J$. It is routine to check that these define a compatible right action and left action of $G$ on $J^{*}$ with right orbits $\lambda G$ and left orbits $G \lambda$. Also, we have two-sided orbits $G \lambda G$ for $\lambda \in J^{*}$. In addition, there is a conjugation action of $G$ on $J^{*}$ defined by $\lambda \mapsto g^{-1} \lambda g$. (The orbits of this conjugation action correspond to the "coadjoint orbits" defined by Kirillov [11] in the case where $G=U_{n}(F)$.)

4.1. Lemma. Let $G=1+J$ be an algebra group. Then the numbers of right orbits of $G$ on $J$ and $J^{*}$ are equal. The same is true about left orbits, two-sided orbits and conjugation orbits.

Proof. More generally, let $G$ be an arbitrary finite group that acts (on the right and linearly) on a finite $F$-vector space $V$. If we let $G$ act on the dual space $V^{*}$ according to the formula $(\lambda g)(v)=\lambda\left(v g^{-1}\right)$, we show that $G$ has equal numbers of orbits in its actions on $V$ and $V^{*}$. By the well known orbit-counting formula often (erroneously) attributed to Burnside, the number of orbits of $G$ in an arbitrary action is the average number of fixed points of the elements of $G$. It suffices, therefore, to show that each element $g \in G$ fixes equal numbers of vectors $v \in V$ and linear functionals $\lambda \in V^{*}$.

The element $g \in G$ fixes $\lambda \in V^{*}$ if and only if $g^{-1}$ fixes $\lambda$, and this happens precisely when $\lambda(v)=\lambda(v g)$ for all $v \in V$. In other words, $g$ fixes $\lambda$ if and only if $v g-v \in \operatorname{ker} \lambda$ for all $v \in V$. The linear functionals fixed by $g$, therefore, are exactly those whose kernel contains $W=\{v g-v \mid v \in V\}$. Because the action of $G$ on $V$ is linear, we see that $W$ is a subspace of $V$, and thus the number of linear functionals fixed by $g$ is exactly $|V| /|W|$.

Now consider the linear transformation $v \mapsto v-v g$ from $V$ onto $W$. Then $|W|=|V| /|K|$, where $K$ is the kernel of this map, and thus $|K|=|V| /|W|$ is the number of $g$-fixed linear functionals. But clearly, $K$ is the set of $g$-fixed vectors, and thus $g$ fixes equal numbers of points in $V$ and $V^{*}$, as desired.

In particular, in the case where $G=1+J$ is an algebra group, this shows that there are equal numbers of right orbits and equal numbers of conjugation orbits of $G$ on $J$ and $J^{*}$. To see that the numbers of left orbits are equal, it suffices to define a new (right) action of $G$ on $J$ by setting $x \cdot g=g^{-1} x$. Finally, to see that 
the numbers of two-sided orbits of $G$ on $J$ and $J^{*}$ are equal, we apply the previous reasoning to the (right) action of $G \times G$ on $J$ defined by $x \cdot(g, h)=g^{-1} x h$. This completes the proof.

Note that we have not proved (and in general, it is not true) that there is a bijection between the right $G$-orbits on $J$ and on $J^{*}$ that preserves orbit sizes. Similarly for the other actions, there is no reason to believe that the sizes of the orbits in $J$ and $J^{*}$ agree.

We next recall a useful bit of elementary linear algebra. If $W$ is an arbitrary finite dimensional vector space with dual space $W^{*}$, then there is a natural map $X \mapsto X^{\perp}=\left\{\lambda \in W^{*} \mid X \subseteq \operatorname{ker} \lambda\right\}$ from the set of subspaces of $W$ to the set of subspaces of $W^{*}$. This map is an order-reversing bijection, and so if $X, Y \subseteq W$, we have $(X \cap Y)^{\perp}=X^{\perp}+Y^{\perp}$. Also, for every subspace $X \subseteq W$, the dimension of its "perpendicular" $X^{\perp}$ is equal to the codimension of $X$.

Given $\lambda \in J^{*}$, define $R_{\lambda}=\{x \in J \mid J x \subseteq \operatorname{ker} \lambda\}$ and note that $R_{\lambda}$ is a subalgebra of $J$, and in fact, it is a left ideal. Similarly, set $L_{\lambda}=\{x \in J \mid x J \subseteq \operatorname{ker} \lambda\}$, and observe that $L_{\lambda}$ is a right ideal. In particular, since $R_{\lambda}$ and $L_{\lambda}$ are subalgebras, we see that $1+R_{\lambda}$ and $1+L_{\lambda}$ are subgroups of $G=1+J$.

4.2. Lemma. Let $G=1+J$ be an algebra group over a field $F$ of order $q$. Fix $\lambda \in J^{*}$ and write

$$
U=\left\{\mu \in J^{*} \mid R_{\lambda} \subseteq \operatorname{ker} \mu\right\} \quad \text { and } \quad V=\left\{\nu \in J^{*} \mid L_{\lambda} \subseteq \operatorname{ker} \nu\right\} .
$$

The following then hold.

(a) $1+R_{\lambda}$ is the right stabilizer of $\lambda$ in $G$ and $|\lambda G|=|J| /\left|R_{\lambda}\right|$ is a power of $q$.

(b) $1+L_{\lambda}$ is the left stabilizer of $\lambda$ in $G$ and $|G \lambda|=|J| /\left|L_{\lambda}\right|$ is a power of $q$.

(c) $\lambda G=\lambda+V$.

(d) $G \lambda=\lambda+U$.

(e) $|\lambda G|=|G \lambda|$ and $\left|R_{\lambda}\right|=\left|L_{\lambda}\right|$.

Proof. Let $g \in G$ and write $g^{-1}=1+t$ with $t \in J$. If $x \in J$ is arbitrary, then

$$
(\lambda g)(x)=\lambda\left(x g^{-1}\right)=\lambda(x+x t)=\lambda(x)+\lambda(x t),
$$

and thus $\lambda$ and $\lambda g$ agree at $x$ precisely when $x t \in$ ker $\lambda$. In particular, $\lambda=\lambda g$ if and only if $J t \subseteq \operatorname{ker} \lambda$, or equivalently, $t \in R_{\lambda}$. It follows that the right stabilizer of $\lambda$ in $G$ is $1+R_{\lambda}$, and thus $|\lambda G|=|G| /\left|1+R_{\lambda}\right|=|J| /\left|R_{\lambda}\right|$. This number is a power of $q=|F|$ since $R_{\lambda}$ is a subalgebra. This proves (a), and the proof of (b) is similar.

To prove (c), we again let $g \in G$ and write $g^{-1}=1+t$. If $x \in L_{\lambda}$, then $x t \in \operatorname{ker} \lambda$ and the previous computation shows that $(\lambda g-\lambda)(x)=\lambda(x t)=0$. In other words, $\lambda g-\lambda \in V$, and hence $\lambda G$ is contained in the additive coset $\lambda+V$. If follows that $|\lambda G| \leq|V|=|J| /\left|L_{\lambda}\right|=|G \lambda|$. The reverse containment follows by similar reasoning, and thus we have equality throughout. This proves (e) and (c); the proof of $(\mathrm{d})$ is similar.

Given $\lambda$ in $J^{*}$, let $D_{\lambda}=R_{\lambda} \cap L_{\lambda}$, and write $n_{\lambda}=\left|R_{\lambda}\right| /\left|D_{\lambda}\right|=\left|L_{\lambda}\right| /\left|D_{\lambda}\right|$. (Note that these two quotients are equal by Lemma $4.2(\mathrm{e})$. Note also that $n_{\lambda}$ is a power of $q=|F|$ since both $R_{\lambda}$ and $D_{\lambda}$ are subalgebras.)

4.3. Lemma. Let $G=1+J$ be an algebra group over a field $F$ of order $q$, and fix $\lambda \in J^{*}$. Then the two-sided orbit $G \lambda G$ of $\lambda$ is the union of $n_{\lambda}$ left orbits that are transitively permuted by the right action of $G$, and it is also the union of $n_{\lambda}$ 
right orbits that are transitively permuted by the left action of $G$. Furthermore, $|G \lambda G|=|J| /\left|D_{\lambda}\right|$ is a power of $q$ and $n_{\lambda}$ is a power of $q$.

Proof. By Lemma 4.2, we have $\lambda G=\lambda+V$ and $G \lambda=\lambda+U$, and thus

$$
|\lambda G \cap G \lambda|=|(\lambda+V) \cap(\lambda+U)|=|\lambda+(V \cap U)|=|V \cap U| .
$$

By Lemma 3.1, therefore,

$$
|G \lambda G|=\frac{|\lambda G||G \lambda|}{|\lambda G \cap G \lambda|}=\frac{|V||U|}{|V \cap U|}=|V+U| .
$$

We know, however, that

$$
V+U=\left(L_{\lambda}\right)^{\perp}+\left(R_{\lambda}\right)^{\perp}=\left(L_{\lambda} \cap R_{\lambda}\right)^{\perp}=\left(D_{\lambda}\right)^{\perp},
$$

and thus $V+U$ has dimension equal to the codimension of $D_{\lambda}$. This yields $|G \lambda G|=$ $|V+U|=|J| /\left|D_{\lambda}\right|$, as claimed. This is clearly a power of $q$, and we have already seen that $n_{\lambda}$ is a power of $q$.

Now $G \lambda G$ is the union of the right translates of the left orbit $G \lambda$. Each of these right translates is a left orbit, and so the distinct right translates of $G \lambda$ are disjoint, and they partition $G \lambda G$. Since these translates all have equal cardinality, their number is

$$
\frac{|G \lambda G|}{|G \lambda|}=\frac{|J| /\left|D_{\lambda}\right|}{|J| /\left|L_{\lambda}\right|}=\frac{\left|L_{\lambda}\right|}{\left|D_{\lambda}\right|}=n_{\lambda} .
$$

Of course, a similar argument works with "right" and "left" interchanged, and the proof is complete.

For completeness, we also discuss the sizes of the conjugation orbits (i.e. coadjoint orbits) in $J^{*}$. We shall not need this, however, for our study of the supercharacters of algebra groups. Fix $\lambda \in J^{*}$ and write $[x, y]=x y-y x$ for elements $x, y \in J$. Define an alternating $F$-bilinear form $\langle\cdot, \cdot\rangle$ on $J$ by setting $\langle x, y\rangle=\lambda([x, y])$, and let $T_{\lambda}$ be the radical of this form. Recall that by definition, $T_{\lambda}=\{x \in J \mid\langle x, J\rangle=0\}=\{y \in J \mid\langle J, y\rangle=0\}$, and in particular, $T_{\lambda}$ is a subspace of $J$. As is well known, the codimension of the radical of an alternating form is always even, and thus $|J| /\left|T_{\lambda}\right|$ is a power of $q=|F|$ with even exponent.

4.4. Lemma. Let $G=1+J$ be an algebra group over a field $F$ of order $q$. Fix $\lambda \in J^{*}$ and let $\mathcal{O}$ be its conjugation orbit. Then $T_{\lambda}$ is a subalgebra and $1+T_{\lambda}$ is the stabilizer of $\lambda$ in the conjugation action. Also, $|\mathcal{O}|=|J| /\left|T_{\lambda}\right|$ is a power of $q$ with even exponent.

Proof. Let $g \in G$ and write $g^{-1}=1+t$ with $t \in J$. Given $x \in J$, we have

$$
(g \lambda-\lambda g)(x)=\lambda\left(g^{-1} x-x g^{-1}\right)=\lambda(t x-x t)=\langle t, x\rangle .
$$

It follows that $g \lambda=\lambda g$ if and only if $\langle t, J\rangle=0$, or equivalently, $t \in T_{\lambda}$. In other words, the conjugation stabilizer of $\lambda$ in $G$ is exactly $1+T_{\lambda}$. But this stabilizer is a subgroup, and thus if $s, t \in T_{\lambda}$, then $(1+s)(1+t)$ is in the stabilizer, and hence $s+t+s t \in T_{\lambda}$. We know, however, that $T_{\lambda}$ is a subspace, and it follows that st $\in T_{\lambda}$ and $T_{\lambda}$ is a subalgebra. Now $|\mathcal{O}|=|G| /\left|1+T_{\lambda}\right|=|J| /\left|T_{\lambda}\right|$, and we have seen that this number is a power of $q$ with even exponent.

We can now obtain an analog of Theorem 3.3.

4.5. Theorem. Let $G=1+J$ be an algebra group. Let $\lambda \in J^{*}$ and suppose that $L_{\lambda}+R_{\lambda}=J$. Then $G \lambda G$ is the conjugation orbit of $\lambda$. 
Proof. We know that the conjugation orbit of $\lambda$ is contained in $G \lambda G$ and has size $|J| /\left|T_{\lambda}\right|$. Since $|G \lambda G|=|J| /\left|D_{\lambda}\right|$ by Lemma 4.3 , it suffices to show that $|J| /\left|T_{\lambda}\right| \geq|J| /\left|D_{\lambda}\right|$, or equivalently, that $\left|T_{\lambda}\right| \leq\left|D_{\lambda}\right|$. In fact, we show that $T_{\lambda} \subseteq D_{\lambda}$. (We mention that the reverse containment is always true, and is easy to see, but this is irrelevant.)

Let $t \in T_{\lambda}$ and $x \in J$, and write $x=l+r$, where $l \in L_{\lambda}$ and $r \in R_{\lambda}$. We compute that

$$
\lambda(t x)=\lambda(t r+t l)=\lambda(t l)=\lambda(l t)=0,
$$

where the second equality holds because $r \in R_{\lambda}$, the third holds because $t \in T_{\lambda}$ and the fourth holds because $l \in L_{\lambda}$. Also, because $t \in T_{\lambda}$, we have $\lambda(x t)=\lambda(t x)=0$. Since $x \in J$ was arbitrary, it follows that $t J \subseteq \operatorname{ker} \lambda$ and $J t \subseteq \operatorname{ker} \lambda$. In other words, $t \in L_{\lambda} \cap R_{\lambda}=D_{\lambda}$, as required.

By Lemma 4.1, we know that the number of conjugation orbits of $G$ on $J^{*}$ is equal to the the number of conjugation orbits of $G$ on $J$, which, of course, is the number of conjugacy classes of $G$. Each conjugation orbit of $G$ on $J^{*}$ has square size by Lemma 4.4, and clearly, the sum of these orbit sizes equals $\left|J^{*}\right|=|J|=|G|$.

Consider the list of integers obtained by taking the square roots of the sizes of the conjugation orbits of $G$ on $J^{*}$. These numbers are powers of $q$; the sum of their squares is $|G|$, and the length of the list is the number of classes of $G$. In other words, this list of square roots of orbit sizes resembles the list of degrees of the irreducible characters of $G$. In an unpublished set of notes [7] circulated by Isaacs in 1997, these numbers were called the "fake character degrees" of the algebra group $G$, and it was suggested that perhaps they are always the actual irreducible character degrees.

In the case where $J^{p}=0$, where $p$ is the characteristic of $F$, one can mimic an argument of Kazhdan for upper triangular groups to prove that the fake character degrees are the true character degrees. Indeed, one can actually construct an irreducible character (with the correct degree) associated with each conjugation orbit of linear functionals. (This is a variation on Kirillov's approach in [11]; we will say more about it later.) Proofs were given by Isaacs [7] in his unpublished 1997 notes, and also by André [2] and (with extensions and improvements) by J. Sangroniz [13].

If $J^{p} \neq 0$, then in general, Kirillov's method fails, and the functions it defines are not necessarily characters. Nevertheless, computer experiments suggested that perhaps the fake degrees are always the actual degrees, even in this case. But recently, a counterexample was constructed by A. Jaikin-Zapirain [10]. It is not always true that the fake degrees are the actual degrees.

\section{Complex values and characters}

Given an algebra group $G=1+J$ over the finite field $F$, we want to use the $F$-valued linear functionals on $J$ to construct complex-valued functions on $G$. To do this, we fix a nontrivial homomorphism $\alpha \mapsto \widetilde{\alpha}$ from the additive group of $F$ into the multiplicative group of the complex numbers $\mathbb{C}$. In other words, this map is any one of the $q-1$ nonprincipal linear characters of the additive group $F^{+}$of $F$, where $q=|F|$.

For each linear functional $\lambda \in J^{*}$, we write $\tilde{\lambda}: J \rightarrow \mathbb{C}$ to denote the function defined by $\widetilde{\lambda}(x)=\widetilde{\lambda(x)}$, for $x \in J$. Thus $\widetilde{\lambda}$ is a linear character of the additive 
group $J^{+}$of $J$. Also, it is clear that the map $\lambda \mapsto \widetilde{\lambda}$ is a homomorphism from the additive group of $J^{*}$ into the multiplicative group of linear characters of $J^{+}$.

5.1. Lemma. Let $J$ be a finite dimensional F-space. Then the map $\lambda \mapsto \widetilde{\lambda}$ is an isomorphism from $\left(J^{*}\right)^{+}$onto the group of linear characters of $J^{+}$.

Proof. Since $\left|J^{*}\right|=|J|=\left|\operatorname{Irr}\left(J^{+}\right)\right|$, it suffices to check that the kernel of our homomorphism is trivial. This is clear, however, since if $\lambda \in J^{*}$ is nonzero, then $\lambda(J)=F$, and thus $\lambda(J)$ cannot be contained in the kernel of the fixed nonprincipal linear character $\alpha \mapsto \widetilde{\alpha}$ on $F^{+}$.

Our various orbit decompositions of $J^{*}$ can be transferred via the isomorphism $\lambda \mapsto \widetilde{\lambda}$ to decompositions of the group of linear characters of $J^{+}$. It is reassuring to observe that the resulting decompositions of $\operatorname{Irr}\left(J^{+}\right)$are not at all dependent on the choice of the nontrivial linear character $\alpha \mapsto \widetilde{\alpha}$ of $F^{+}$. To see why this is so, observe that if $\gamma \in F$ is arbitrary, then the map $\alpha \mapsto \widetilde{\gamma \alpha}$ is a linear character of $F^{+}$, and as $\gamma$ runs over the elements of $F$, these run over all of the $q$ different linear characters of $F^{+}$. Changing our original linear character of $F^{+}$, therefore, is equivalent to multiplication by some nonzero scalar $\gamma \in F$. This has no effect on the orbit decompositions because all of our actions are $F$-linear.

Given the algebra group $G=1+J$, the map $g \mapsto g-1$ defines a natural bijection from $G$ to $J$. We can use this map to convert functions defined on $J$ to functions defined on $G$. Specifically, if $\lambda \in J^{*}$, then $\widetilde{\lambda}$ is defined on $J$, and this, in turn, determines a complex-valued function $\widehat{\lambda}$ on $G$ via the formula $\widehat{\lambda}(g)=\widetilde{\lambda}(g-1)$. We mention for future use that $\widehat{-\lambda}$ is the complex conjugate of the function $\widehat{\lambda}$.

Recall that if $H$ is an arbitrary finite group, there is a standard inner product

$$
[\alpha, \beta]=\frac{1}{|H|} \sum_{h \in H} \alpha(h) \overline{\beta(h)}
$$

defined for complex-valued functions on $H$. Also, the irreducible characters of $H$ form an orthonormal set with respect to this inner product. In particular, the functions $\tilde{\lambda}$ on $J$ are orthonormal, and since $J^{+}$is abelian, these functions form a basis for the space of all complex valued functions on $J$. It follows that the functions $\hat{\lambda}$ on $G=1+J$ form an orthonormal basis for the space of all complexvalued functions on $G$. Of course, these functions are not generally characters (or even class functions) on $G$. It is clear, however, that $\widehat{\lambda}(1)=1$ for all $\lambda \in J^{*}$.

Although our principal interest will be in the functions obtained by summing $\hat{\lambda}$ as $\lambda$ runs over a two-sided $G$-orbit on $J^{*}$, we digress briefly to discuss sums over conjugation orbits.

5.2. Lemma. Let $G=1+J$ be an algebra group and let $\lambda \in J^{*}$. Then

$$
\widehat{g^{-1} \lambda g}(h)=\widehat{\lambda}\left(g h g^{-1}\right)
$$

for elements $g, h \in G$. In particular, the sum of the functions $\hat{\lambda}$ as $\lambda$ runs over a conjugation orbit in $J^{*}$ is constant on conjugacy classes of $G$.

Proof. Write $h=1+t$ with $t \in J$ and observe that $g h g^{-1}=1+g t g^{-1}$. We have

$$
\widehat{g^{-1} \lambda g}(h)=\widehat{g^{-1} \lambda g}(t)=\widetilde{\lambda}\left(g t g^{-1}\right)=\widehat{\lambda}\left(g h g^{-1}\right),
$$

as wanted. The second assertion is clear. 
Given a conjugation orbit $\mathcal{O}$ in $J^{*}$, define the function $\psi_{\mathcal{O}}$ on $G$ by

$$
\psi_{\mathcal{O}}=\frac{1}{\sqrt{|\mathcal{O}|}} \sum_{\lambda \in \mathcal{O}} \hat{\lambda}
$$

Note that $\psi_{\mathcal{O}}$ is a class function, that $\left[\psi_{\mathcal{O}}, \psi_{\mathcal{O}}\right]=1$ and that the "degree" $\psi_{\mathcal{O}}(1)=$ $\sqrt{|\mathcal{O}|}$ is a positive integer. (This last assertion follows from Lemma 4.4.) Also, as $\mathcal{O}$ runs over the various conjugation orbits, the functions $\psi_{\mathcal{O}}$ are orthogonal. Furthermore, by Lemma 4.1, the number of these functions is the number of conjugacy classes of $G$. Also, the sum of the squares of their degrees is the sum of the sizes of the conjugation orbits on $J^{*}$, which, of course, equals $\left|J^{*}\right|=|J|=|G|$. We shall refer to these functions $\psi_{\mathcal{O}}$ as the Kirillov functions of the algebra group $G$.

It was conjectured by Kirillov [11] that in the case where $G=U_{n}(F)$, the unimodular upper triangular group, these Kirillov functions are exactly the irreducible characters of $G$. It is now known, however, that this cannot be correct in general. This is because the Kirillov functions always have values in the cyclotomic field of $p$ th roots of unity, but irreducible characters of upper triangular groups can have values that are not in this field. (For example, if $p=2$, the group $U_{13}(F)$ has a nonreal irreducible character. See [8] and [9].) Nevertheless, as we shall see in Corollary 5.11, many of the Kirillov functions for the groups $U_{n}(F)$ are actually irreducible characters, even when $n$ is large when compared to $p$.

We mentioned earlier that if $J^{p}=0$, where $p$ is the characteristic of $F$, then a variation on Kirillov's method does successfully construct the irreducible characters of the algebra group $G=1+J$. The trick here is to replace the bijection $g \mapsto g-1$ from $G$ onto $J$ by another bijection: $g \mapsto \ln (g)$, where the "natural logarithm" is defined by the appropriate truncated power series. (For details, see [13].)

Since the two-sided $G$-orbits in $J^{*}$ are unions of conjugation orbits, it follows that the functions obtained by summing $\widehat{\mu}$ for $\mu \in G \lambda G$ are class functions. In fact, we will see that these sums are characters, and we will show that they always have the form $\sigma_{X}=\sum_{\chi \in X} \chi(1) \chi$ for some subset $X \subseteq \operatorname{Irr}(G)$.

Fix an algebra group $G=1+J$, and consider the complex group algebra $\mathbb{C} G$, whose elements are the formal $\mathbb{C}$-linear combinations of the elements of $G$. There is a natural vector-space isomorphism between $\mathbb{C} G$ and the $\mathbb{C}$-space $M$ of complex-valued functions on $G$ : the function $\alpha: G \rightarrow \mathbb{C}$ corresponds to the element $\sum_{g \in G} \alpha(g) g$. Of course, $G$ acts on $\mathbb{C} G$ by left and right multiplication, and it is easy to check that the corresponding left and right actions of $G$ on $M$ are defined by the formulas

$$
(f g)(h)=f\left(h g^{-1}\right) \quad \text { and } \quad(g f)(h)=f\left(g^{-1} h\right)
$$

for $f \in M$ and $g, h \in G$. With these actions, therefore, $M$ is isomorphic to $\mathbb{C} G$ as a $G$-bimodule.

We have seen that $M$ has a natural basis: the functions $\hat{\lambda}$ for $\lambda \in J^{*}$. We investigate the behavior of these basis functions under left and right action by group elements.

5.3. Lemma. Let $G=1+J$ be an algebra group, and let $M$ be the space of complex valued functions on $G$, as above. Then for $\lambda \in J^{*}$ and $g \in G$, we have

$$
\widehat{\lambda} g=\widehat{\lambda}\left(g^{-1}\right) \widehat{\lambda g} \quad \text { and } \quad g \widehat{\lambda}=\widehat{\lambda}\left(g^{-1}\right) \widehat{g \lambda} .
$$


In particular if $Y \subseteq J^{*}$ is a left, right or two-sided $G$-invariant set, then the linear span $U_{Y}$ of the functions $\widehat{\mu}$ for $\mu \in Y$ is a left, right or two-sided G-invariant subspace of $M$, respectively.

Proof. Let $h \in G$. We compute

$$
\begin{aligned}
(\widehat{\lambda} g)(h) & =\widehat{\lambda}\left(h g^{-1}\right) \\
& =\widetilde{\lambda}\left(h g^{-1}-1\right) \\
& =\widetilde{\lambda}\left(\left(g^{-1}-1\right)+(h-1) g^{-1}\right) \\
& =\widetilde{\lambda}\left(g^{-1}-1\right) \widetilde{\lambda}\left((h-1) g^{-1}\right) \\
& =\widehat{\lambda}\left(g^{-1}\right) \widetilde{\lambda g}(h-1) \\
& =\widehat{\lambda}\left(g^{-1}\right) \widehat{\lambda g}(h),
\end{aligned}
$$

as wanted. The computation for the left action is similar. The last assertion should now be clear.

Under the natural isomorphism between $M$ and $\mathbb{C} G$, we see that if $Y \subseteq J^{*}$ is left $G$-invariant, right $G$-invariant or two-sided $G$-invariant, then the subspace $U_{Y} \subseteq M$ corresponds to a left ideal, a right ideal or a two-sided ideal, respectively, of the group algebra $\mathbb{C} G$.

We can now give the principal definition of this paper. Let $\lambda \in J^{*}$ and let $Y=(-\lambda) G$ be the right $G$-orbit of $-\lambda$. By Lemma 5.3 , the space $U_{Y}$ spanned by the functions $\widehat{\mu}$ for $\mu \in Y$ is a right module for $G$, and hence it affords a character of $G$, which we call $\chi_{\lambda}$. We refer to these characters as the supercharacters of $G$, but of course, we still need to reconcile this with the terminology of Section 2 . From the definition, we see that $\chi_{\lambda}(1)=\operatorname{dim}\left(U_{Y}\right)=|Y|=|\lambda G|$.

It may seem odd that we have chosen notation so that $\chi_{\lambda}$ is constructed using the orbit of the negative of $\lambda$, rather than $\lambda$ itself. The reason for this is that by doing so, we obtain simpler formulas for the character $\chi_{\lambda}$. For example, if $\lambda$ happens to be right- $G$-invariant, we will see that $\hat{\lambda}$ is a linear character of $G$, and in this case, $\chi_{\lambda}=\widehat{\lambda}$.

More generally, if $\lambda \in J^{*}$ is arbitrary, we know by Lemma 4.2 that the subgroup $H=1+R_{\lambda}$ is the right stabilizer of $\lambda$ in $G$. We have the following.

5.4. Theorem. Let $G=1+J$ and let $\lambda \in J^{*}$. Then the restriction $\tau$ of $\widehat{\lambda}$ to $H=1+R_{\lambda}$ is a linear character of $H$, and $\chi_{\lambda}=\tau^{G}$, the induced character.

Proof. Let $Y=-\lambda G$ and let $U_{Y}$ be the linear span of the functions $\widehat{\mu}$ for $\mu \in Y$. These functions $\widehat{\mu}$ form a basis for $U_{Y}$, which, therefore, can be written as the direct sum of the one-dimensional subspaces $\mathbb{C} \widehat{\mu}$ for $\mu \in Y$. By Lemma 5.3, moreover, these spaces are transitively permuted by the right action of $G$. (The transitivity follows from the fact that $Y$ is a right $G$-orbit.)

Now fix $\mu=-\lambda$. Since the stabilizer of the space $\mathbb{C} \widehat{\mu}$ is $H$, it follows that the character $\chi_{\lambda}$ afforded by $U_{Y}$ is induced from the linear character of $H$ corresponding to the action of $H$ on the one-dimensional space $\mathbb{C} \widehat{\mu}$. For $h \in H$, however, Lemma 5.3 yields

$$
\widehat{\mu} h=\widehat{\mu}\left(h^{-1}\right) \widehat{\mu h}=\widehat{\mu}\left(h^{-1}\right) \widehat{\mu} .
$$

In particular, the function $h \mapsto \widehat{\mu}\left(h^{-1}\right)$ defines a linear character of $H$, and so $\widehat{\mu}\left(h^{-1}\right)$ is the complex conjugate of $\widehat{\mu}(h)$. But since $\mu=-\lambda$, we see that $\widehat{\mu}(h)$ is 
the complex conjugate of $\hat{\lambda}(h)=\tau(h)$. Thus $\tau$ is a linear character of $H$, and it is afforded by the right action of $H$ on $\mathbb{C} \widehat{\mu}$. In particular, $\chi_{\lambda}=\tau^{G}$, as wanted.

We mention that an easy calculation can be used to show directly that the function $\tau$ is a linear character of $H$.

Our next result gives several significant properties of the supercharacters of an algebra group. Before stating it, however, we recall some relevant notation. First, if $\lambda \in J^{*}$, then $n_{\lambda}$ is the number of right $G$-orbits (and also the number of left $G$-orbits) in the two-sided orbit $G \lambda G$. (See Lemma 4.3 and the definition of $n_{\lambda}$ preceding it.) Also, if $X \subseteq \operatorname{Irr}(G)$ is an arbitrary subset, then $\sigma_{X}=\sum_{\chi \in X} \chi(1) \chi$. Recall also that each (two-sided) ideal $I$ of a group algebra $\mathbb{C} G$ is the direct sum of the minimal ideals that it contains. The minimal ideals, moreover, correspond to the irreducible characters of $G$, and if $X$ is the set of irreducible characters corresponding to minimal ideals contained in the ideal $I$, then $I$, viewed as a right $\mathbb{C} G$-module, affords the character $\sigma_{X}$.

5.5. Theorem. Let $G=1+J$ and let $\lambda \in J^{*}$. The following then hold.

(a) $\chi_{\lambda}$ depends only on the two-sided orbit $G \lambda G$ containing $\lambda$.

(b) If $X \subseteq \operatorname{Irr}(G)$ is the set of irreducible constituents of $\chi_{\lambda}$, then $n_{\lambda} \chi_{\lambda}=\sigma_{X}$.

(c) Supercharacters corresponding to distinct two-sided $G$-orbits in $J^{*}$ have disjoint sets of irreducible constituents, and these sets partition $\operatorname{Irr}(G)$.

Recall that in the general supercharacter theory of Section 2, we had a partition $\mathcal{X}$ of $\operatorname{Irr}(G)$, and for each member $X \in \mathcal{X}$, the associated supercharacter was the function $\sigma_{X}$. We mentioned, however, that a constant multiple $c_{X} \sigma_{X}$ would work as well, and so our choice of $c_{X}=1$ was somewhat arbitrary. Of course, since we would like supercharacters to be actual characters, there are some restrictions on the constants $c_{X}$. Specifically, since the multiplicity of a character $\psi \in X$ as a constituent of $\sigma_{X}$ is $\psi(1)$, we want $c_{X} \psi(1)$ to be a positive integer for all $\psi \in X$.

By Theorem 5.5(b), we see that for algebra groups, we have $\chi_{\lambda}=\left(1 / n_{\lambda}\right) \sigma_{X}$, and so we have $c_{X}=1 / n_{\lambda}$, where $X$ is the set of irreducible constituents of $\chi_{\lambda}$. By construction, $\chi_{\lambda}$ is a character, and hence the requirement that $c_{X} \psi(1)$ is a positive integer for $\psi \in X$ is automatically satisfied.

Proof of Theorem 5.5. If $T \subseteq J^{*}$, then following our previous notation, we write $U_{T}$ to denote the linear span in $M$ of the functions $\widehat{\mu}$ for $\mu \in T$. (Recall that $M$ is the space of all complex-valued functions on $G$.) Write $Y=-\lambda G$, as before, and let $Z=-G \lambda G$, so that $Y$ and $Z$ are the sets of negatives of the members of $\lambda G$ and $G \lambda G$, respectively. Then $Z$ is the disjoint union of $n_{\lambda}$ left translates of $Y$, and hence $U_{Z}$ is the direct sum of $n_{\lambda}$ subspaces of the form $U_{(g Y)}$ for $g \in G$. By Lemma 5.3, however, we see that $U_{(g Y)}=g U_{Y}$, and so as right $G$-modules, the summands of $U_{Z}$ are all isomorphic. It follows that the character afforded by $U_{Z}$ (viewed as a right $G$-module) is exactly $n_{\lambda} \chi_{\lambda}$.

Now $U_{Z}$ is both left and right $G$-invariant by Lemma 5.3 , and so under the natural bimodule isomorphism $M \cong \mathbb{C} G$, the subspace $U_{Z}$ corresponds to a (twosided) ideal of $\mathbb{C} G$. It follows that the character $n_{\lambda} \chi_{\lambda}$ afforded by $U_{Z}$ has the form $\sigma_{X}$ for some subset $X \subseteq \operatorname{Irr}(G)$. This establishes both (a) and (b).

As $J^{*}$ is the disjoint union of the two-sided $G$-orbits, it follows that $M$ is the direct sum of subspaces like $U_{Z}$ for the various supercharacters $\chi_{\mu}$. Also, since $M$ affords the regular character $\rho_{G}$ of $G$, it follows that $\rho_{G}$ is the sum of the 
characters $n_{\mu} \chi_{\mu}$ as $\mu$ runs over a set of representatives for the two-sided $G$-orbits in $J^{*}$. Writing $X_{\mu}$ to denote the set of irreducible constituents of $\chi_{\mu}$, we have

$$
\sum_{\mu} \sigma_{X_{\mu}}=\rho_{G}
$$

The multiplicity of each irreducible constituent of one of the characters $\sigma_{X_{\mu}}$ is equal to its degree, which is also equal to its multiplicity in $\rho_{G}$, and so we see that the sets $X_{\mu}$ are disjoint and that they cover $\operatorname{Irr}(G)$.

We now know that the number of different supercharacters of $G=1+J$ is equal to the number of two-sided $G$-orbits on $J^{*}$. By Lemma 4.1, this is equal to the number of two-sided $G$-orbits on $J$, which, in turn, is the number of superclasses of $G$. To establish that we have a genuine supercharacter theory, as in Section 2, only one thing remains: we must show that supercharacters are constant on superclasses. To prove that, we need a convenient way to compute the values of a supercharacter.

5.6. Theorem. Let $G=1+J$ be an algebra group, and let $\lambda \in J^{*}$. Then

$$
\chi_{\lambda}=\frac{1}{n_{\lambda}} \sum_{\mu \in G \lambda G} \widehat{\mu} .
$$

We need a preliminary lemma for the proof of Theorem 5.6.

5.7. Lemma. Let $G=1+J$ be an algebra group. Suppose that $g \in G$ and $\lambda \in J^{*}$ with $\lambda g \neq \lambda$. Then $g$ fixes no member of the left orbit $G \lambda$ and

$$
\sum_{\tau \in G \lambda} \widehat{\tau}(g)=0
$$

Proof. If $(h \lambda) g=h \lambda$ for some element $h \in G$, then it follows via left multiplication by $h^{-1}$ that $\lambda g=\lambda$, which is not the case. In other words, $g$ fixes no member of $G \lambda$, as claimed.

By Lemma 4.2, we have $G \lambda=\lambda+U$, where $U \subseteq J^{*}$ is the subspace consisting of the linear functionals $\mu$ such that $R_{\lambda} \subseteq \operatorname{ker} \mu$. If $\tau=\lambda+\mu$, then $\widehat{\tau}(g)=\widehat{\lambda}(g) \widehat{\mu}(g)$, and hence

$$
\sum_{\tau \in G \lambda} \widehat{\tau}(g)=\widehat{\lambda}(g) \sum_{\mu \in U} \widehat{\mu}(g) .
$$

To complete the proof, therefore, it suffices to show that $\sum \widehat{\mu}(g)=0$, where $\mu$ runs over $U$. Write $g=1+t$ with $t \in J$, and recall that by definition $\widehat{\mu}(g)=\widetilde{\mu}(t)$.

Now consider the "evaluation" linear functional $\mu \mapsto \mu(t)$ on $U$. Since $\lambda g \neq \lambda$, we know that $t \notin R_{\lambda}$. But

$$
R_{\lambda}=\bigcap_{\mu \in U} \operatorname{ker} \mu
$$

and thus evaluation at $t$ is not the zero map on $U$. It follows that the evaluation map is a homomorphism from $U$ onto $F$, and hence each element of $F$ occurs equally often as $\mu(t)$ for $\mu \in U$. If the common multiplicity is $m$, we can write

$$
\sum_{\mu \in U} \widehat{\mu}(g)=\sum_{\mu \in U} \widetilde{\mu}(t)=m \sum_{\alpha \in F} \widetilde{\alpha}=0,
$$

where the final equality holds by orthogonality, because the map $\alpha \mapsto \widetilde{\alpha}$ is a nonprincipal linear character of $F^{+}$. This completes the proof. 
Proof of Theorem 5.6. We have seen that $n_{\lambda} \chi_{\lambda}$ is the character afforded by the right $G$-module $U_{Z}$, which is the linear span of the functions $\widehat{-\mu}$, as $\mu$ runs over $G \lambda G$. We must show that this character is equal to $\sum \widehat{\mu}$, where the sum runs over $\mu \in G \lambda G$.

Fix $g \in G$, and recall that the functions $\widehat{-\mu}$ for $\mu \in G \lambda G$ form a basis for $U_{Z}$. By Lemma 5.3, each of these basis vectors is mapped by $g$ to a scalar multiple of the basis vector $\widehat{-\mu g}$, where the scalar is equal to $-\mu\left(g^{-1}\right)$. It follows that the basis vector $\widehat{-\mu}$ contributes to the trace of the linear transformation of $U_{Z}$ induced by right multiplication by $g$ only when $\mu g=\mu$. When this happens, the contribution to the trace equals $\widehat{-\mu}\left(g^{-1}\right)$. It follows that

$$
n_{\lambda} \chi_{\lambda}(g)=\sum_{\substack{\mu \in G \lambda G \\ \mu g=\mu}} \widehat{-\mu}\left(g^{-1}\right) .
$$

Since $\chi_{\lambda}$ is a character, the complex conjugate of $\chi_{\lambda}(g)$ is $\chi_{\lambda}\left(g^{-1}\right)$, and also, we have seen that the complex conjugate of the function $\widehat{-\mu}$ is $\widehat{\mu}$. If we take complex conjugates of both sides of the above equation, therefore, we obtain

$$
n_{\lambda} \chi_{\lambda}\left(g^{-1}\right)=\sum_{\substack{\mu \in G \lambda G \\ \mu g=\mu}} \widehat{\mu}\left(g^{-1}\right) .
$$

Now, since $g$ fixes $\mu$ if and only if $g^{-1}$ fixes $\mu$, we can substitute $g^{-1}$ for $g$ and write

$$
n_{\lambda} \chi_{\lambda}(g)=\sum_{\substack{\mu \in G \lambda G \\ \mu g=\mu}} \widehat{\mu}(g)
$$

for all elements $g \in G$.

Now consider the "missing" terms of this sum, corresponding to functionals $\mu \in G \lambda G$ that are not fixed by $g$. Since $G \lambda G$ is invariant under the left action of $G$, it follows by Lemma 5.7 that the set of missing linear functionals is a union of left $G$-orbits, and that the sum of $\widehat{\mu}(g)$ over each of these orbits vanishes. It follows that we can add the missing terms to the sum without changing its value, and thus

$$
n_{\lambda} \chi_{\lambda}(g)=\sum_{\mu \in G \lambda G} \widehat{\mu}(g),
$$

as required.

Next, we provide a formula that relates supercharacters and superclasses. Recall that by definition, the superclass containing an element $g=1+t$ of $G=1+J$ is the set $K=1+G t G$. Recall also that if $\chi_{\lambda}$ is a supercharacter, then $\chi_{\lambda}(1)=|\lambda G|$.

5.8. Theorem. Let $G=1+J$ be an algebra group and let $\chi_{\lambda}$ be one of its supercharacters, where $\lambda \in J^{*}$. If $g \in G$ lies in the superclass $K$, then

$$
\chi_{\lambda}(g)=\frac{|\lambda G|}{|G t G|} \sum_{x \in G t G} \tilde{\lambda}(x)=\frac{\chi_{\lambda}(1)}{|K|} \sum_{h \in K} \widehat{\lambda}(h),
$$

where $g=1+t$. In particular, the supercharacters of $G$ are constant on the superclasses.

Proof. The second equality is clear since $K=1+G t G$ and $\chi_{\lambda}(1)=|\lambda G|$, and we recall that $\widehat{\lambda}(1+x)=\widetilde{\lambda}(x)$. Also, the last assertion follows since the sum on the right depends only on the superclass $K$. 
To prove the first equality, consider the map $(u, v) \mapsto u t v$ from $G \times G$ to $G t G$. This map is clearly surjective, and it is easy to see that all elements of $G t G$ are hit equally often. Each element of $G t G$, therefore, has the form utv for exactly $|G|^{2} /|G t G|$ ordered pairs $(u, v)$, and it follows that

$$
\sum_{u, v \in G} \tilde{\lambda}(u t v)=\frac{|G|^{2}}{|G t G|} \sum_{x \in G t G} \tilde{\lambda}(x) .
$$

Similarly, if we map $G \times G$ to $G \lambda G$ via $(u, v) \mapsto u \lambda v$, we see that each element of $G \lambda G$ is hit exactly $|G|^{2} /|G \lambda G|$ times, and this yields

$$
\sum_{u, v \in G} \widetilde{u \lambda v}(t)=\frac{|G|^{2}}{|G \lambda G|} \sum_{\mu \in G \lambda G} \widetilde{\mu}(t)
$$

The left sides of the previous two equations are equal, however, and we deduce that

$$
\frac{1}{|G \lambda G|} \sum_{\mu \in G \lambda G} \tilde{\mu}(t)=\frac{1}{|G t G|} \sum_{x \in G t G} \tilde{\lambda}(x) .
$$

Since $\widehat{\mu}(g)=\widetilde{\mu}(t)$ and $n_{\lambda}=|G \lambda G| /|\lambda G|$, it follows that

$$
\chi_{\lambda}(g)=\frac{1}{n_{\lambda}} \sum_{\mu \in G \lambda G} \widehat{\mu}(g)=\frac{|G \lambda G|}{n_{\lambda}|G t G|} \sum_{x \in G t G} \tilde{\lambda}(x)=\frac{|\lambda G|}{|G t G|} \sum_{x \in G t G} \tilde{\lambda}(x),
$$

as required.

In particular, we now know that the supercharacters and superclasses of an algebra group form a genuine supercharacter theory in the sense of Section 2.

When does it happen that the supercharacter $\chi_{\lambda}$ is irreducible?

5.9. Lemma. Let $G=1+J$ be an algebra group, and let $\chi_{\lambda}$ be one of its supercharacters. Then $\left[\chi_{\lambda}, \chi_{\lambda}\right]=\chi_{\lambda}(1) / n_{\lambda}$.

Proof. For an arbitrary subset $X \subseteq \operatorname{Irr}(G)$, we have

$$
\sigma_{X}(1)=\sum_{\psi \in X} \psi(1)^{2}=\left[\sigma_{X}, \sigma_{X}\right] .
$$

Now $n_{\lambda} \chi_{\lambda}=\sigma_{X}$ for some subset $X \subseteq \operatorname{Irr}(G)$, and thus

$$
n_{\lambda}^{2}\left[\chi_{\lambda}, \chi_{\lambda}\right]=\left[\sigma_{X}, \sigma_{X}\right]=\sigma_{X}(1)=n_{\lambda} \chi_{\lambda}(1),
$$

and the result follows.

5.10. Theorem. Let $G=1+J$ be an algebra group, and let $\lambda \in J^{*}$. Then the supercharacter $\chi_{\lambda}$ is irreducible if and only if $L_{\lambda}+R_{\lambda}=J$. In this case, the twosided orbit $G \lambda G$ is exactly the conjugation orbit of $\lambda$, and the character $\chi_{\lambda}$ is equal to the Kirillov function for this orbit.

Proof. By Lemma 5.9, we have $\left[\chi_{\lambda}, \chi_{\lambda}\right]=\chi_{\lambda}(1) / n_{\lambda}$, and so the character $\chi_{\lambda}$ is irreducible if and only if $\chi_{\lambda}(1)=n_{\lambda}$. Now $\chi_{\lambda}(1)=|\lambda G|=|J| /\left|R_{\lambda}\right|$ and $n_{\lambda}=$ $\left|R_{\lambda}\right| /\left|D_{\lambda}\right|$, where we recall that $D_{\lambda}=R_{\lambda} \cap L_{\lambda}$. Thus $\chi_{\lambda}$ is irreducible if and only if $|J| /\left|R_{\lambda}\right|=\left|R_{\lambda}\right| /\left|D_{\lambda}\right|$. Since $\left|R_{\lambda}\right|=\left|L_{\lambda}\right|$, this is equivalent to $|J|=\left|R_{\lambda}\right|\left|L_{\lambda}\right| /\left|D_{\lambda}\right|$. But the right side of this equation is equal to $\left|L_{\lambda}+R_{\lambda}\right|$, and thus $\chi_{\lambda}$ is irreducible if and only if $L_{\lambda}+R_{\lambda}=J$, as claimed.

Suppose now that $\chi_{\lambda}$ is irreducible. Then $L_{\lambda}+R_{\lambda}=J$, and so Theorem 4.5 tells us that $G \lambda G$ is the conjugation orbit of $\lambda$. Also, $|G \lambda G|=|J| /\left|D_{\lambda}\right|$, and in this case, 
where $L_{\lambda}+R_{\lambda}=J$, this is equal to $\left(\left|R_{\lambda}\right| /\left|D_{\lambda}\right|\right)^{2}=n_{\lambda}^{2}$. Thus $\chi_{\lambda}=\left(1 / n_{\lambda}\right) \sum \widehat{\mu}$ is exactly the Kirillov function corresponding to the conjugation orbit $G \lambda G$.

We can apply Theorem 5.10 to the situation studied by André and Yan: the unimodular upper triangular groups. Let $G=U_{n}(F)=1+J$, where as usual, $J$ is the algebra of strictly upper triangular matrices. For each matrix position $(i, j)$ with $i<j$, let $\lambda_{i, j}$ be the element of $J^{*}$ whose value at a matrix $x \in J$ is the $(i, j)$-entry of $x$.

5.11. Corollary. Let $G=U_{n}(F)=1+J$ as above, and let $\lambda=a \lambda_{i, j}$, where $0 \neq a \in F$ and $1 \leq i<j \leq n$. Then the supercharacter $\chi_{\lambda}$ is irreducible, and it is thus equal to the Kirillov function $\psi_{\mathcal{O}}$, where $\mathcal{O}$ is the conjugation orbit of $\lambda$.

Versions of Corollary 5.11 appear in the work of André and Yan. In fact, in the case where $J=U_{n}(F)$, they have simple combinatorial descriptions of exactly which supercharacters are irreducible. In his paper, Yan refers to a linear functional of the form $a \lambda_{i, j}$ as being "primary", and he observes, in particular, that if $\lambda$ is primary, then the corresponding supercharacter is irreducible. The fact that the corresponding Kirillov character is irreducible appears as Lemma 2 of [3], where André calls these characters "elementary".

Proof of Corollary 5.11. By Theorem 5.10, it suffices to show that $L_{\lambda}+R_{\lambda}=J$. It is enough, therefore, to show that a matrix unit $e_{u, v}$ in $J$ lies either in $R_{\lambda}$ or in $L_{\lambda}$ (or both). To show that $e_{u, v} \in R_{\lambda}$, we must show that $J e_{u, v} \subseteq$ ker $\lambda$, and for this purpose, it suffices to check that $\lambda\left(e_{s, t} e_{u, v}\right)=0$ for all matrix units $e_{s, t}$ in $J$. Similarly, of course, to show that $e_{u, v} \in L_{\lambda}$, we must check that $\lambda\left(e_{u, v} e_{s, t}\right)=0$ for all matrix units $e_{s, t}$ in $J$.

Since $\lambda(x)$ is a multiple of the $(i, j)$-entry of a matrix $x \in J$, we see that if $\lambda\left(e_{s, t} e_{u, v}\right) \neq 0$, we must have $v=j$. Similarly, if $\lambda\left(e_{u, v} e_{s, t}\right) \neq 0$, then $u=i$. This shows that with the possible exception of $e_{i, j}$, every matrix unit $e_{u, v} \in J$ lies in $R_{\lambda} \cup L_{\lambda}$. Since $s<t$, we see that $\lambda\left(e_{s, t} e_{i, j}\right)=0$, and thus $e_{i, j}$ lies in $R_{\lambda}$ (and similarly, it lies in $L_{\lambda}$, too). This completes the proof.

Observe that Corollary 5.11 can be viewed as dual to Corollary 3.4, and like that corollary, it has a natural extension to pattern groups, with essentially no change in the proof.

We close this section with an observation about supercharacters that have linear constituents. Of course, such supercharacters exist since every irreducible character of $G=1+J$ is a constituent of some (unique) supercharacter $\chi_{\lambda}$.

5.12. Corollary. Let $G=1+J$ be an algebra group, and suppose that $\lambda \in J^{*}$. If the supercharacter $\chi_{\lambda}$ has a linear constituent, then $G \lambda=G \lambda G=\lambda G$ and $R_{\lambda}=L_{\lambda}$ is an ideal of $J$.

Proof. We know that $n_{\lambda} \chi_{\lambda}=\sigma_{X}$ for some subset $X \subseteq \operatorname{Irr}(G)$. Since the multiplicity of each irreducible constituent of $\sigma_{X}$ is equal to its degree, it follows that $n_{\lambda}$ divides the degree of every irreducible constituent of $\chi_{\lambda}$. Since there is a linear constituent, we must have $n_{\lambda}=1$. But $n_{\lambda}$ is the number of left orbits in $G \lambda G$, and it is also the number of right orbits in this set, and it follows that $G \lambda=G \lambda G=\lambda G$, as claimed.

In general, $n_{\lambda}=\left|R_{\lambda}\right| /\left|D_{\lambda}\right|=\left|L_{\lambda}\right| /\left|D_{\lambda}\right|$, and so in our case, $R_{\lambda}=D_{\lambda}=L_{\lambda}$. Since $L_{\lambda}$ is a right ideal and $R_{\lambda}$ is a left ideal, the result now follows. 
Before we close this section, we give a brief summary of its principal results. Given $\lambda \in J^{*}$, we defined the supercharacter $\chi_{\lambda}$ as the character afforded by the $G$-module constructed as the linear span of the functions $\widehat{\mu}$, as $\mu$ runs over $-\lambda G$, and we saw that $\chi_{\lambda}$ is induced from a linear character of an algebra subgroup of $G$. We showed that $\chi_{\lambda}$ depends only on the two-sided $G$-orbit containing $\lambda$, and that $\chi_{\lambda}=\left(1 / n_{\lambda}\right) \sum \widehat{\mu}$, where $\mu$ runs over $G \lambda G$. Also, $\chi_{\lambda}$ is constant on the superclass $1+G x G$, for $x \in J$.

We showed that as $\lambda$ runs over a set of representatives for the two-sided $G$ orbits on $J^{*}$, the sets $X_{\lambda}$ of irreducible constituents of $\chi_{\lambda}$ partition $\operatorname{Irr}(G)$, and that together with the partition of $G$ into superclasses, they form a supercharacter theory in the sense of Section 2. Also, $\chi_{\lambda}=\left(1 / n_{\lambda}\right) \sigma_{X_{\lambda}}$. Finally, necessary and sufficient conditions for $\chi_{\lambda}$ to be irreducible are given, and connections with the Kirillov orbit method are discussed.

\section{Restriction, induCtion AND PRODUCtS}

Let $G=1+J$ be an algebra group. If $A \subseteq J$ is a subalgebra, then $H=1+A$ is a subgroup of $G$ that is itself an algebra group. Our first goal in this section is to show that in this situation, the restriction to $H$ of a supercharacter of $G$ must be a nonnegative integer linear combination of supercharacters of $H$. We need the following fact, which appears as Lemma 3.1 in [6].

6.1. Lemma. Let $J$ be a nilpotent algebra, and suppose that $A \subseteq J$ is a subalgebra. If $J^{2}+A=J$, then $A=J$.

Proof. First, we observe that since $A^{n} \subseteq A$, we have $J^{n}=\left(A+J^{2}\right)^{n} \subseteq A+J^{n+1}$ for $n \geq 1$, and thus $A+J^{n} \subseteq A+J^{n+1}$. The reverse containment is clear, and so we have

$$
J=A+J^{2}=A+J^{3}=A+J^{4}+\cdots .
$$

The result now follows since $J^{n}=0$ for sufficiently large $n$.

6.2. Corollary. Let $A$ be a maximal subalgebra of the nilpotent algebra $J$. Then $A$ contains $J^{2}$, and $A$ is an ideal of $J$ with codimension 1 .

Proof. Since $A<J$, Lemma 6.1 yields $A \subseteq A+J^{2}<J$. But $J^{2}$ is an ideal, and hence $A+J^{2}$ is a subalgebra. Thus $A=A+J^{2}$ by the maximality of $A$, and in particular, $A \supseteq J^{2}$. Every subspace of $J$ containing $J^{2}$ is an ideal, however, and thus $A$ is an ideal and is a maximal subspace of $J$. The result follows.

Next, we investigate the restriction map $J^{*} \rightarrow A^{*}$, where $A \subseteq J$ is a subalgebra of codimension 1 .

6.3. Lemma. Let $G=1+J$ be an algebra group over a field $F$ of order $q$, and let $X \subseteq J^{*}$ be a two-sided $G$-orbit. Suppose that $A \subseteq J$ is a subalgebra of codimension 1 , and let $Y \subseteq A^{*}$ be the image of $X$ under the restriction map from $J^{*}$ to $A^{*}$. The following then hold.

(a) There is an integer $r \in\{1, q\}$ such that the preimage in $X$ of each member of $Y$ has cardinality $r$.

(b) If either $L_{\lambda} \subseteq A$ or $R_{\lambda} \subseteq A$ for some member $\lambda \in X$, then then $r=q$.

(c) If there exists $\lambda \in X$ such that neither $L_{\lambda} \subseteq A$ nor $R_{\lambda} \subseteq A$, then $Y$ is a two-sided $H$-orbit, and the image in $Y$ of each right or left $G$-orbit in $X$ is respectively a right or left $H$-orbit. 
Proof. Let $W=\left\{\tau \in J^{*} \mid A \subseteq \operatorname{ker} \tau\right\}$, and note that $|W|=q$. Since $J^{2} \subseteq A \subseteq \operatorname{ker} \tau$ by Corollary 6.2 , we have $L_{\tau}=J=R_{\tau}$ for $\tau \in W$, and it follows by Lemma 4.2 that each member of $W$ is both left and right $G$-invariant.

If $\lambda \in X$, then $\lambda+W$ is the full set of elements in $J^{*}$ that have the same restriction to $A$ as $\lambda$. The preimage in $X$ of the restriction of $\lambda$ to $A$ is thus $\lambda+W_{\lambda}$, where $W_{\lambda}=\{\tau \in W \mid \lambda+\tau \in X\}$. To prove (a), therefore, we must show that the sets $W_{\lambda}$ all have equal cardinality $r$ for $\lambda \in X$, and that $r$ is either 1 or $q$.

We claim that in fact, the sets $W_{\lambda}$ are all equal. To see this, let $\lambda \in X$ and $g, h \in G$. If $\tau \in W$, then $g \lambda h+\tau=g \lambda h+g \tau h=g(\lambda+\tau) h$, and this linear functional lies in $X$ if and only if $\lambda+\tau$ lies in $X$. In other words $W_{\lambda}=W_{g \lambda h}$, as claimed.

Recall that in Lemma 4.2, we defined $U=\left\{\mu \in J^{*} \mid R_{\lambda} \subseteq \operatorname{ker} \mu\right\}$. If $R_{\lambda} \subseteq A$ for some member $\lambda \in X$, then $W \subseteq U$. By Lemma 4.2, however, $\lambda+U=G \lambda \subseteq X$, and thus $W_{\lambda}=W$ in this case. Thus $r=|W|=q$, and a similar argument shows that $r=q$ if $L_{\lambda} \subseteq A$. This proves (b) and part of (a).

Now suppose that for some member $\lambda \in X$, both $L_{\lambda} \nsubseteq \subseteq A$ and $R_{\lambda} \nsubseteq A$. Then $A \cap R_{\lambda}$ and $A \cap L_{\lambda}$ have codimension 1 in $R_{\lambda}$ and $A_{\lambda}$, respectively. It follows by Lemma 4.2 that the right and left stabilizers of $\lambda$ in $H=1+A$ have index $q$ in the corresponding stabilizers in $G$. But $H$ has index $q$ in $G$, and therefore, the indices in $H$ of the right and left stabilizers of $\lambda$ in $H$ are equal to the indices in $G$ of the right and left stabilizers of $\lambda$ in $G$. It follows that $\lambda G=\lambda H$ and $G \lambda=H \lambda$, and we conclude that $G \lambda G=H \lambda H$. In this situation, we see that $Y$, which is the restriction to $A$ of $X=G \lambda G=H \lambda H$, is a two-sided $H$-orbit in $A^{*}$. Also, the restrictions of the one-sided orbits in $X$ are one-sided orbits in $Y$, and this proves (c).

Finally, we observe that $r=|X| /|Y|$. In this case, both $X$ and $Y$ are two-sided orbits (of $G$ and $H$ ), and so each of $|X|$ and $|Y|$ is a power of $q$, and thus $r$ is a power of $q$. (See Lemma 4.3.) We know, however, that $r \leq|W|=q$, and so there are just two possibilities: either $r=1$ or $r=q$. This completes the proof of (a).

We are now ready to prove the main result of this section.

6.4. Theorem. Let $G=1+J$ be an algebra group over the field $F$ of order $q$. Let $A \subseteq J$ be an arbitrary subalgebra and write $H=1+A$. Then the restriction to $H$ of each supercharacter of $G$ is a nonnegative integer linear combination of supercharacters of $H$.

Proof. We can assume without loss that $A$ is a maximal subalgebra of $J$, and thus by Corollary 6.2 , it has codimension 1 . Let $\lambda \in J^{*}$ be arbitrary and consider the restriction of the supercharacter $\chi_{\lambda}$ to $H=1+A$.

Recall that by Lemma 6.3, the number $r$ of members of $X=G \lambda G$ with restriction equal to the restriction of $\lambda$ is independent of the choice $\lambda$ in $X$. Also, either $r=1$ or $r=q$.

By Theorem 5.6, we know that $n_{\lambda} \chi_{\lambda}$ is the sum of the functions $\widehat{\mu}$, as $\mu$ runs over the two-sided orbit $X$. Since the restriction of $\widehat{\mu}$ to $H$ is $\widehat{\nu}$, where $\nu$ is the restriction of $\mu$ to $A$, it follows that $\left(n_{\lambda} \chi_{\lambda}\right)_{H}$ is $r$ times the sum of the functions $\widehat{\nu}$ on $H$, where $\nu$ runs over the image $Y$ of $X$ under restriction to $A$. Thus

$$
\left(\chi_{\lambda}\right)_{H}=\frac{r}{n_{\lambda}} \sum_{\nu \in Y} \widehat{\nu} .
$$


As $X$ is both left and right $H$-invariant, $Y$ is a union of two-sided $H$ orbits. It follows that $\left(\chi_{\lambda}\right)_{H}$ is a nonnegative rational linear combination of supercharacters of $H$, where for $\nu \in Y$, the coefficient of the corresponding supercharacter of $H$ is $n_{\nu} r / n_{\lambda}$. Our goal, therefore, is to show that this is an integer for every choice of $\nu \in Y$.

Since $r$ and $n_{\lambda}$ are independent of $\lambda \in X$, we can, if necessary, replace $\lambda$ by some member of $X$ with restriction equal to $\nu$, and so we assume that $\nu$ is the restriction of $\lambda$ to $A$. Write $L=L_{\lambda}$ and $R=R_{\lambda}$ and let $L_{1}$ and $R_{1}$ respectively be $L_{\nu}$ and $R_{\nu}$, computed in $A$. Let $D=L \cap R$ and $D_{1}=L_{1} \cap R_{1}$. By definition, then, $n_{\lambda}=|L| /|D|$ and $n_{\nu}=\left|L_{1}\right| /\left|D_{1}\right|$, and so

$$
\frac{n_{\nu}}{n_{\lambda}}=\frac{\left|L_{1}\right| /\left|D_{1}\right|}{|L| /|D|}=\frac{\left|L_{1}\right| /|L|}{\left|D_{1}\right| /|D|} .
$$

We also consider the subspace $L_{0}=\{x \in J \mid x A \subseteq$ ker $\lambda\}$, and we observe that $L \subseteq L_{0}$ and $A \cap L_{0}=L_{1}$.

Suppose now that $R \subseteq A$, and note that in this case, $R \subseteq R_{1}$ and $r=q$. We argue that $L$ has codimension 1 in $L_{0}$. To see this, consider the bilinear form $\langle\cdot, \cdot\rangle$, defined on $J$ by the formula $\langle x, y\rangle=\lambda(x y)$. This form establishes a nondegenerate bilinear pairing between $J / L$ and $J / R$, and we observe that $L_{0}$ is the "left perpendicular" of $A$. Since $R \subseteq A \subseteq J$, it follows that the codimension of $L$ in $L_{0}$ is equal to the codimension of $A$ in $J$, and this is 1 , as wanted.

Now $L_{1}=L_{0} \cap A$ has codimension at most 1 in $L_{0}$, and so there are two possibilities: either the codimension is 1 and $|L|=\left|L_{1}\right|$, or the codimension is 0 and $L_{0}=L_{1} \subseteq A$. Also, recall that $|L|=|R|$ and $\left|L_{1}\right|=\left|R_{1}\right|$ by Lemma 4.2. If $|L|=\left|L_{1}\right|$, therefore, then $|R|=\left|R_{1}\right|$, and thus $R=R_{1}$. In this case, $D=R \cap L$ and $D_{1}=R \cap L_{1}$, and since both $L$ and $L_{1}$ have codimension 1 in $L_{0}$, it follows that $L \cap L_{1}$ has codimension at most 1 in each of $L$ and $L_{1}$, and thus $D \cap D_{1}$ has codimension at most 1 in each of $D$ and $D_{1}$. Since $|L|=\left|L_{1}\right|$ in this case, it follows from equation $(*)$ that $n_{\nu} / n_{\lambda}=|D| /\left|D_{1}\right|$ lies in the set $\{1 / q, 1, q\}$. Since $r=q$ in this case, $r n_{\nu} / n_{\lambda}$ is in the set $\left\{1, q, q^{2}\right\}$, and so it is an integer, as required.

Now suppose that $L_{0}=L_{1}$. Then $L \subseteq L_{1}$, and the codimensions of $L$ and $R$ in $L_{1}$ and $R_{1}$, respectively, are 1 . It follows that the codimension of $D$ in $D_{1}$ is at most 2 , and in this case, $\left|L_{1}\right| /|L|=q$. Again, we see that $n_{\nu} / n_{\lambda}$ lies in the set $\{1 / q, 1, q\}$, and again $r n_{\nu} / n_{\lambda}$ is in the set $\left\{1, q, q^{2}\right\}$.

We can now assume that $R \nsubseteq A$, and similarly, we can assume that $L \nsubseteq A$. Then Lemma 6.3(c) applies, and so the image of the restriction map from $G \lambda G$ into $A^{*}$ is $H \nu H$ and the image of $\lambda G$ is $\nu H$. Since the preimage of each member of $H \nu H$ in $G \lambda G$ has cardinality $r$, we see that

$$
\frac{|G \lambda G|}{|H \nu H|}=r=\frac{|\lambda G|}{|\nu H|} .
$$

Thus

$$
n_{\lambda}=\frac{|G \lambda G|}{|\lambda G|}=\frac{|H \nu H|}{|\nu H|}=n_{\nu},
$$

and in this case $n_{\lambda} / n_{\nu}=1$. Thus $r n_{\lambda} / n_{\mu}=r \in\{1, q\}$, and the proof is complete.

Next, we begin working toward a proof that the product of two supercharacters of an algebra group is always a nonnegative integer linear combination of supercharacters. We know that the supercharacters of $G$ form a basis for the space of 
complex-valued functions on $G$ that are constant on superclasses, and so it is clear that a product of two supercharacters is a $\mathbb{C}$-linear combination of supercharacters. In fact, it is easy to see that the coefficients must be nonnegative rational numbers. This is because the multiplicity of $\chi_{\lambda}$ in the product $\pi$ is $[\pi, \psi] /\left[\chi_{\lambda}, \psi\right]$, where $\psi$ is any irreducible constituent of $\chi_{\lambda}$. (This works because $\psi$ is a constituent of just one supercharacter.) To prove that the coefficients are actually integers, however, requires a more subtle argument. Our strategy is to consider direct products.

Let $K$ and $L$ be finite dimensional nilpotent algebras over the same field $F$, and consider the external direct sum $M=K \oplus L$, where we view $K$ and $L$ as ideals of $M$. In particular $K$ and $L$ are subalgebras of $M$, and so $X=1+K$ and $Y=1+L$ are algebra subgroups of $G=1+M$, and clearly, $X \cap Y=1$. Also, since $K L=0=L K$, we see that $(1+k)(1+l)=1+k+l=(1+l)(1+k)$ for $k \in K$ and $l \in L$, and it follows that $X Y=G$ and that $X$ and $Y$ centralize each other. In particular, $G$ is the direct product of its subgroups $X$ and $Y$.

Now if $\alpha$ and $\beta$ are respectively characters of $X$ and $Y$, then $\alpha \times \beta$ is the function on $G$ defined by the formula $(\alpha \times \beta)(x y)=\alpha(x) \beta(y)$, and it is a standard fact that $\alpha \times \beta$ is a character of $G$. Somewhat analogously, if $\lambda \in K^{*}$ and $\mu \in L^{*}$, we can define a linear functional $\lambda \oplus \mu$ on $M$ by defining $(\lambda \oplus \mu)(k+l)=\lambda(k)+\mu(l)$. Furthermore, it is not hard to see that every member of $M^{*}$ is uniquely of the form $\lambda \oplus \mu$ for $\lambda \in K^{*}$ and $\mu \in L^{*}$.

6.5. Lemma. Assume the above notation. Suppose that $\alpha$ and $\beta$ are supercharacters of $X=1+K$ and $Y=1+L$ corresponding respectively to linear functionals $\lambda \in K^{*}$ and $\mu \in L^{*}$. Then $\alpha \times \beta$ is the supercharacter $\chi_{\nu}$ of $G=1+M$, where $\nu=\lambda \oplus \mu$.

Proof. First, we observe that if $x \in X$ and $y \in Y$, then $\nu x y=\lambda x \oplus \mu y$, and similarly for left actions. It follows that we can write $\nu G=\lambda X \oplus \mu Y$ and $G \nu G=$ $X \lambda X \oplus Y \mu Y$. We therefore have

$$
n_{\nu}=\frac{|G \nu G|}{|\nu G|}=\frac{|X \lambda X||Y \mu Y|}{|\lambda X||\mu Y|}=n_{\lambda} n_{\mu}
$$

where, of course, $n_{\lambda}$ and $n_{\mu}$ are computed in the algebra groups $X=1+K$ and $Y=1+L$, respectively.

Now write $x=1+k$ and $y=1+l$, where $k \in K$ and $l \in L$, and recall that $x y=1+k+l$. We have

$$
\widehat{\nu}(x y)=\widetilde{\nu}(k+l)=\widetilde{\lambda}(k) \widetilde{\mu}(l)=\widehat{\lambda}(x) \widehat{\mu}(y)=(\widehat{\lambda} \times \widehat{\mu})(x y),
$$

and thus we can write $\widehat{\nu}=\widehat{\mu} \times \widehat{\nu}$. Of course, a similar decomposition holds for all members of $G \nu G=X \mu X \oplus Y \nu Y$. Now

$$
n_{\nu} \chi_{\nu}=\sum_{\delta \in G \nu G} \widehat{\delta}=\left(\sum_{\sigma \in X \mu X} \widehat{\sigma}\right) \times\left(\sum_{\tau \in Y \mu Y} \widehat{\tau}\right)=\left(n_{\lambda} \alpha\right) \times\left(n_{\mu} \beta\right),
$$

and the result follows.

6.6. Theorem. Let $G=1+J$ be an algebra group and let $\alpha$ and $\beta$ be supercharacters of $G$. Then $\alpha \beta$ is a nonnegative integer linear combination of supercharacters of $G$.

Proof. Applying the preceding theory with $K=J=L$, we conclude that $\alpha \times \beta$ is a supercharacter of the algebra group $G \times G=1+(J \oplus J)$. To avoid confusion, we view the elements of $J \oplus J$ as ordered pairs of elements of $J$, and similarly, we 
view the elements of $G \times G$ as ordered pairs of elements of $G$. In particular, we have $1+(x, y)=(1+x, 1+y)$, for $x, y \in J$.

Now consider the diagonal subalgebra $D=\{(x, x) \mid x \in J\}$, and note that $D$ really is a subalgebra of $J \oplus J$, and in fact, $D \cong J$. Let $H=1+D$ be the corresponding algebra subgroup of $G \times G$, and observe that $H$ is the diagonal subgroup of $G \times G$ consisting of the elements of the form $(g, g)$, with $g \in G$. Then $H \cong G$, and if we identify $H$ with $G$ via the natural isomorphism, we see that the restriction of the supercharacter $\alpha \times \beta$ to $H$ is just the ordinary product character $\alpha \beta$. Since $H=1+D$ is an algebra subgroup, however, Theorem 6.4 applies, and we conclude that $\alpha \beta$ is a nonnegative integer linear combination of supercharacters.

Finally, we discuss induction. We have shown that the restriction of a supercharacter of an algebra group to an algebra subgroup is always a nonnegative integer linear combination of supercharacters, and so it is tempting to guess that the analogous property also holds for induction. This is wrong, however; the character induced by a supercharacter of an algebra subgroup need not even be a superclass function. (In other words, it may not be constant on superclasses.) To see why this is so, recall that every superclass function on an algebra group is a linear combination of supercharacters, and so if induction were to map supercharacters of algebra subgroups to superclass functions, it would also map arbitrary superclass functions to superclass functions. It is easy, however, to construct a counterexample to this. Let $J$ be the $F$-algebra with basis $\{x, y, z\}$, where $x y=z=y x$ and all other products of basis vectors are zero. Then $H=1+F x$ is an algebra subgroup, and the element $1+x$ constitutes a full superclass of $H$. Define $\varphi$ on $H$ by setting $\varphi(1+x)=1$ and $\varphi(h)=0$ for all other elements $h \in H$, and observe that $\varphi$ is indeed a superclass function. Since $G=1+J$ is abelian we see that $1+x$ is the only element of $G$ on which the induced function $\varphi^{G}$ is nonzero. But $1+z$ and $1+x$ are in the same superclass of $G$, and hence $\varphi^{G}$ is not a superclass function.

It is possible to modify the definition of induction in algebra groups so that the result of "inducing" a superclass function of an algebra subgroup is always a superclass function. Furthermore, this can be done so that the analog of Frobenius reciprocity is valid. But as we shall see, even with this modified induction, it is not generally true that supercharacters of an algebra subgroup yield integer linear combinations of supercharacters of the whole group.

Let $H=1+A$ be an algebra subgroup of $G=1+J$, and let $\varphi$ be an arbitrary complex-valued function of $H$. Extend $\varphi$ to the function $\varphi^{0}$ on $G$ by setting $\varphi^{0}(g)=$ 0 if $g \notin H$. We now define the superinduced function $\varphi^{(G)}$ on $G$ by the formula

$$
\varphi^{(G)}(1+x)=\frac{1}{|G||H|} \sum_{u, v \in G} \varphi^{0}(1+u x v)
$$

for $x \in J$. Even with no assumption on $\varphi$, it is immediate that the superinduced function $\varphi^{(G)}$ is a superclass function on $G$. Observe that if $x=0$, the above formula becomes

$$
\varphi^{(G)}(1)=\frac{|G|^{2}}{|G||H|} \varphi(1)=|G: H| \varphi(1) .
$$

The multiplicative constant $1 /(|G||H|)$ in our definition of superinduction may seem rather arbitrary, but it is exactly what is needed so that the promised analog of Frobenius reciprocity holds. 
6.7. Lemma. Let $H=1+A$ be an algebra subgroup of $G=1+J$ and suppose that $\varphi$ is a function defined on $H$ and that $\theta$ is a superclass function of $G$. Then $\left[\varphi^{(G)}, \theta\right]=\left[\varphi, \theta_{H}\right]$.

Proof. We have

$$
\left[\varphi^{(G)}, \theta\right]=\frac{1}{|G|} \sum_{g \in G} \varphi^{(G)}(g) \overline{\theta(g)}=\frac{1}{|G|} \frac{1}{|G||H|} \sum_{\substack{t \in J \\ u, v \in G}} \varphi^{0}(1+u t v) \overline{\theta(1+t)} .
$$

Now write $s=u t v$ and observe that by the definition of $\varphi^{0}$, we need to consider only elements $s \in A$. Also, since $\theta$ is a superclass function, we have $\theta(1+t)=$ $\theta\left(1+u^{-1} s v^{-1}\right)=\theta(1+s)$, and thus

$$
\left[\varphi^{(G)}, \theta\right]=\frac{1}{|G|^{2}|H|} \sum_{\substack{s \in A \\ u, v \in G}} \varphi^{0}(1+s) \overline{\theta(1+s)}=\frac{1}{|H|} \sum_{s \in A} \varphi(1+s) \overline{\theta(1+s)}=\left[\varphi, \theta_{H}\right]
$$

as wanted.

In the situation of Lemma 6.7, suppose that $\varphi$ is a supercharacter of $H$ and that $\theta$ is a supercharacter of $G$. Then $\varphi$ occurs with integer multiplicity in $\theta_{H}$, and so $\left[\varphi_{H}, \theta\right]$ is an integer, and in fact, it is a multiple of $[\varphi, \varphi]$. It follows by Lemma 6.7 that $\left[\varphi^{(G)}, \theta\right]$ is a multiple of $[\varphi, \varphi]$, but unless we know that it is a multiple of $[\theta, \theta]$, it does not follow that $\theta$ occurs with integer multiplicity in $\varphi^{(G)}$. This argument does show, however, that when the superclass function $\varphi^{(G)}$ is written as a linear combination of supercharacters of $G$, all of the coefficients are nonnegative rational numbers.

Indeed, the coefficients may not be integers, as is shown in the example we are about to sketch. Let $J$ have basis $\{x, r, l, z\}$ over the field $F$ of order 2 , and suppose that $x^{2}=r+l+z, r x=z, x l=z$ and all other products of basis vectors are zero. (It is not hard to show that this really does define a nilpotent algebra.) Let $A=F r+F l+F z$, and note that the product of every two elements of $A$ is zero. Thus $H=1+A$ is an algebra subgroup of $G=1+J$, and all supercharacters of $H$ are linear. For every such supercharacter $\varphi$, therefore, we have $\varphi^{(G)}(1)=|G: H| \varphi(1)=2$. We will produce a supercharacter $\theta$ of $G$ of degree 4 , and thus if $\varphi$ occurs with positive coefficient in the decomposition of $\theta_{H}$, it will follow that $\theta$ occurs with a positive nonintegral coefficient in $\varphi^{(G)}$.

Let $\lambda \in J^{*}$ be the linear functional with kernel $K=F x+F r+F l$. It is easy to check that $R_{\lambda}=F r+F z$, and so $\chi_{\lambda}(1)=|\lambda G|=|J| /\left|R_{\lambda}\right|=4$. This provides the counterexample we seek.

Appendix A: The work of André and Yan. As we explained in Section 1, many of the results of this paper are generalizations to arbitrary finite algebra groups of theorems of André and Yan about the unimodular upper triangular groups $U_{n}(F)$. André began his study of the "basic characters" of $U_{n}(F)$ in his Ph.D. thesis in 1992, and he developed the theory in a series of papers in the Journal of Algebra. (The basic characters defined by André are exactly our supercharacters in his situation.) André constructed his basic characters as certain products of "elementary" characters, which are the irreducible characters described in our Corollary 5.11.

Originally, André's work relied on a construction due to Kazhdan of the irreducible characters of $G=U_{n}(F)$ in the case where the characteristic $p$ of $F$ satisfies $p \geq n$. Under that assumption, there exist inverse maps exp : $J \rightarrow G$ and 
$\ln : G \rightarrow J$, where $J$ is the algebra of strictly upper triangular $n \times n$ matrices over $F$. Using these maps, Kazhdan was able to associate an irreducible character of $G$ to each coadjoint orbit (conjugation orbit) of $G$ on $J^{*}$. (We mention that an accessible, and more general version of Kazhdan's construction can be found in [6], where Sangroniz works with algebra groups $G=1+J$ such that $J^{p}=0$.)

Later, in [3], André was able to drop the requirement that $p \geq n$. In that paper, he proved directly that his elementary characters are, in fact, irreducible characters, and then, using what we have called "Kirillov functions", and appealing to his earlier work, he was able to reproduce his results on basic characters, but without requiring that $p \geq n$.

Independently, and at approximately the same time, Yan [14] extended André's earlier (that is, $p \geq n$ ) results to the general case. But Yan used an entirely different, and more elementary method; his (unpublished) paper is self-contained, and his arguments are independent of André's. Yan used the left and right actions of $G$ on $J$ and $J^{*}$, as we have done here, and indeed, our paper was inspired by Yan's work, and owes a significant debt to it.

Of course, in the context of specific groups, such as the upper triangular groups $U_{n}(F)$, there is more information than one can hope to prove in general. We devote the rest of this appendix to a description of some of the results of André and Yan that do not seem to have analogs for arbitrary algebra groups.

Let $G=U_{n}(F)=1+J$, where as before, $J$ is the algebra of strictly upper triangular $n \times n$ matrices over $F$. Yan shows that each two-sided $G$-orbit in $J$ contains exactly one matrix with the property that no row and no column contains more than one nonzero entry. The superclasses of $G$ are thus parameterized by the following combinatorial data. First, choose a set $D$ of matrix positions $(i, j)$ such that $1 \leq i<j \leq n$ and no two elements of $D$ agree in either the first or the second coordinate. Next, choose a function $\tau$ from $D$ into the nonzero elements of $F$. Now $D$ and $\tau$ together determine a strictly upper triangular matrix $x=x(D, \tau)$, whose nonzero entries occur exactly at positions $(i, j)$ in $D$, and whose $(i, j)$-entry is $\tau(i, j)$. Each superclass of $G$ contains just one element of the form $1+x(D, \tau)$, and thus the pairs $(D, \tau)$ parameterize the superclasses.

Yan observes that the same combinatorial objects, the pairs $(D, \tau)$ as above, also parameterize the two-sided $G$-orbits on $J^{*}$, and thus they parameterize the supercharacters of $G$. (André parametrizes his characters using the same combinatorial data.) Let $\lambda_{i, j} \in J^{*}$ be the linear functional that picks out the $(i, j)$ entry of each matrix $x \in J$. Then $J^{*}$ is exactly the linear span of the linear functionals $\lambda_{i, j}$. Given $\lambda \in J^{*}$, Yan refers to the set of pairs $(i, j)$ such that $\lambda_{i, j}$ occurs with a nonzero coefficient in the expansion of $\lambda$ as the support of $\lambda$. He shows that each two-sided orbit in $J^{*}$ contains exactly one linear functional $\lambda$ whose support set $D$ has the form described above, and thus one can write

$$
\lambda=\sum_{(i, j) \in D} \tau(i, j) \lambda_{i, j}
$$

where $\tau: D \rightarrow F^{\times}$is as before. Every supercharacter of $G$, therefore, is uniquely of the form $\chi_{\lambda}$, where $\lambda$ is determined by the pair $(D, \tau)$ as above.

Each of the summands $\tau(i, j) \lambda_{(i, j)}$ in the decomposition of $\lambda$ is primary, which means that it is supported at a single position, and hence the supercharacter corresponding to each of these primary summands of $\lambda$ is irreducible. (See Corollary 5.11 
and the paragraph following it.) Yan shows that $\chi_{\lambda}$ is the product of these irreducible supercharacters, and he calls this the "primary decomposition". In particular, every supercharacter of $G=U_{n}(F)$ can be written as a product of irreducible characters, a fact that was used in André's definition of his basic characters.

Yan uses a combinatorial argument, together with his primary decomposition, to prove that products of supercharacters of $U_{n}(F)$ are nonnegative integer linear combinations of supercharacters. Of course, that fact is included in our more general Theorem 6.6, although our proof is (necessarily) quite different.

Appendix B: Connection with Gelfand pairs and spherical functions. Let $G$ be a finite group with subgroup $H \subseteq G$, and let $\pi$ be the permutation character of $G$ acting on the right cosets of $H$. (Thus $\pi=\left(1_{H}\right)^{G}$.) Recall that the pair $(G, H)$ is said to be a Gelfand pair if $\pi$ is multiplicity-free. (See [12] and the references there for more on Gelfand pairs.) In this situation, let $S$ be the set of irreducible constituents of $\pi$. For each character $\chi \in S$, define the corresponding spherical function $s_{\chi}$ on $G$ by setting

$$
s_{\chi}(g)=\frac{1}{|H|} \sum_{h \in H} \chi(g h),
$$

and note that

$$
s_{\chi}(1)=\left[\chi_{H}, 1_{H}\right]=\left[\chi,\left(1_{H}\right)^{G}\right]=[\chi, \pi]=1 .
$$

(To be somewhat more precise, one could say that the functions $s_{\chi}$ are the normalized spherical functions since we have chosen the multiplicative constant $1 /|H|$ so that these functions will have value 1 at the identity of $G$.)

It is clear that if $f$ is one of the spherical functions $s_{\chi}$ and $g \in G$ is arbitrary, then $f(g h)=f(g)=f(h g)$ for all elements $h \in H$, and thus we can say that $f$ is $H$-bi-invariant. In fact, the $|S|$ spherical functions $s_{\chi}$ form an orthogonal basis for the space of all such $H$-bi-invariant functions on $G$.

Now suppose that $A$ is an abelian group acted on by some group $H$, and let $G$ be the semidirect product $A H$. It is not hard to see that $(G, H)$ is a Gelfand pair in this situation, and one can construct the characters in the set $S$ fairly explicitly. It turns out that the restrictions to $A$ of the distinct members of $S$ are exactly the sums of the characters in the various $H$-orbits of irreducible (linear) characters of $A$, and in particular, there is one member of $S$ and one spherical function for each $H$-orbit on $\operatorname{Irr}(A)$. Let $\Lambda$ be such an orbit and let $\chi$ be the corresponding member of $S$. Given an arbitrary element $a h$ of $G$, where $a \in A$ and $h \in H$, one computes that $\chi(a h)$ is exactly the sum of $\mu(a)$ as $\mu$ runs over the members of the orbit $\Lambda$ that happen to be fixed by $h$.

We can now compute the values of the normalized spherical function $s=s_{\chi}$ on $G$, where $\chi$ corresponds to $\Lambda$, as above. First, since $s$ is $H$-bi-invariant, we have $s(a h)=s(a)$, and so it suffices to evaluate $s$ at elements of $A$. For this purpose, we let $\mathcal{P}$ be the set of ordered pairs defined by

$$
\mathcal{P}=\left\{(\mu, h) \mid \mu \in \Lambda, h \in H \text { and } \mu^{h}=\mu\right\} .
$$

We have

$$
s(a)=\frac{1}{|H|} \sum_{h \in H} \chi(a h)=\frac{1}{|H|} \sum_{(\mu, h) \in \mathcal{P}} \mu(a)=\frac{1}{|H|} \sum_{\mu \in \Lambda}\left|H_{\mu}\right| \mu(a),
$$


where $H_{\mu}$ is the stabilizer in $H$ of $\mu$. But $|H| /\left|H_{\mu}\right|=|\Lambda|$ for all $\mu \in \Lambda$, and so

$$
s(a h)=s(a)=\frac{1}{|\Lambda|} \sum_{\mu \in \Lambda} \mu(a)
$$

is the average value of the characters in $\Lambda$ at $a \in A$.

How does this relate to the supercharacters of an algebra group $G=1+J$ ? Let $H=G \times G$ and $A=J$, and let $H$ act on $A$ by $x^{(u, v)}=u^{-1} x v$, where $x \in J$ and $u, v \in G$. The linear characters of $A$ are exactly the functions $\widetilde{\nu}$, for $\nu \in J^{*}$, and the $H$-orbits on $\operatorname{Irr}(A)$ are the sets $\{\widetilde{\nu} \mid \nu \in X\}$, where $X$ is a two-sided orbit in $J^{*}$.

Recall now that $\widehat{\nu}(1+x)=\widetilde{\nu}(x)$ for $\nu \in J^{*}$ and $x \in J$. If $\chi_{\lambda}$ is a supercharacter of $G$, therefore, and $1+x=g \in G$, we have

$$
n_{\lambda} \chi_{\lambda}(g)=\sum_{\nu \in G \lambda G} \widehat{\nu}(g)=\sum_{\nu \in G \lambda G} \widetilde{\nu}(x)=|G \lambda G| s_{\lambda}(x),
$$

where $s_{\lambda}$ is the normalized spherical function on the semidirect product $J(G \times G)$ corresponding to the $(G \times G)$-orbit containing the linear character $\tilde{\lambda}$ of $J$. Since $n_{\lambda}=|G \lambda G| /|\lambda G|$, this yields $\chi_{\lambda}(g)=|\lambda G| s_{\lambda}(x)$.

Appendix C: Some examples. Let $F$ be a finite field and fix a positive integer $n$. Let $R=F[X] /\left(X^{n+1}\right)$, the polynomial ring in the indeterminate $X$ modulo the ideal generated by $X^{n+1}$. Write $x$ to denote the image of $X$ in $R$, and observe that $x^{n+1}=0$ and that every element of $R$ is represented uniquely as a polynomial $f=f(x)$ with degree at most $n$. The radical $J$ of $R$, of course, is the set of these polynomials with zero "constant" term, and $J$ has dimension $n$. Because $J$ is commutative, the two-sided orbits of $G$ on $J$ and on $J^{*}$ are really one-sided orbits, and this simplifies our calculations.

Although one could work more generally, we will take $|F|=2$ for simplicity. First, we determine the superclasses of $G=1+J$. For $1 \leq i \leq n$, it is easy to see that $x^{i} G$ is exactly the set of polynomials in $f$ having low-order term $x^{i}$. The $G$-orbits of nonzero elements of $J$, therefore, are parameterized by the integers in the range $1 \leq i \leq n$, where $f$ is in the orbit with parameter $i$ precisely when $x^{i}$ is the low order term of $f$. Clearly, orbit $i$ has size $2^{n-i}$. The remaining orbit, of course, is $\{0\}$, to which we assign the parameter 0 . Thus $G$ has exactly $n+1$ superclasses, parameterized by the integers $0,1, \ldots, n$. Superclass 0 is $\{1\}$, and for $i>0$, superclass $i$ contains $1+x^{i}$ and has size $2^{n-i}$.

For $1 \leq i \leq n$, let $\lambda_{i} \in J^{*}$ be the linear functional that picks out the coefficient of $x^{i}$ in an element $f \in J$, and observe that $\left\{\lambda_{1}, \lambda_{2}, \ldots, \lambda_{n}\right\}$ is a basis for $J^{*}$. Now let $f \in J$ and write $f=a_{1} x+a_{2} x^{2}+\cdots+a_{n} x^{n}$, with coefficients $a_{i} \in F$. It is easy to compute the action of $G$, and we see that

$$
\left(\lambda_{i}\right)(1+f)=\lambda_{i}+a_{1} \lambda_{i-1}+a_{2} \lambda_{i-2}+\cdots+a_{i-1} \lambda_{1},
$$

and so $\lambda_{i} G$ is the set of all linear combinations of the $\lambda_{j}$ with $j \leq i$, and such that the coefficient of $\lambda_{i}$ is 1 . It follows that the orbits of nonzero members of $J^{*}$ can be parameterized by the integers $1,2, \ldots, n$, where orbit $i$ contains all members $\lambda \in J^{*}$ such that when $\lambda$ is expanded in terms of the $\lambda_{k}$, the largest subscript corresponding to a nonzero coefficient is $i$. The size of orbit $i$, therefore, is $2^{i-1}$. We assign the parameter 0 to the remaining $G$-orbit in $J^{*}$, namely $\{0\}$.

Since the supercharacters of $G$ correspond to the $G$-orbits in $J^{*}$, we can name these characters $\chi_{i}$ with $0 \leq i \leq n$. We know that the supercharacters are constant 
on the superclasses, and so there is an $(n+1) \times(n+1)$ supercharacter table that we want to compute.

In our case, where $J$ is commutative, two-sided orbits are one-sided orbits, and we see that the numbers $n_{\lambda}$ are all equal to 1 . It follows that $\chi_{i}$ is exactly the sum of the functions $\hat{\lambda}$, where $\lambda$ runs over orbit $i$ on $J^{*}$. In particular, $\chi_{i}(1)$ is the size of orbit $i$, and we know that this is 1 if $i=0$ and is $2^{i-1}$ otherwise. Also, if $\lambda=0$, then $\hat{\lambda}$ is the constant function 1 , and thus $\chi_{0}$ is the principal character of $G$.

To complete the determination of the supercharacter table, we compute $\chi_{i}\left(1+x^{j}\right)$, where $i$ and $j$ lie in $\{1,2, \ldots, n\}$. Since $\widehat{\lambda}\left(1+x^{j}\right)=\widetilde{\lambda}\left(x^{j}\right)$, we see that this number is 1 if $\lambda\left(x^{j}\right)=0$ and is -1 if $\lambda\left(x^{j}\right)=1$. If $i<j$, then for all linear functionals $\lambda$ in orbit $i$, we have $\lambda\left(x^{j}\right)=0$, and thus $\chi_{i}\left(1+x^{j}\right)$ is equal to the size of orbit $i$, namely $2^{i-1}$.

Orbit $i$ on $J^{*}$ consists of linear functionals of the form $\lambda_{i}+\mu$, where $\mu$ is a linear combination of the functionals $\lambda_{k}$ for $k<i$. All of the members of orbit $i$, therefore, have value 1 on $x^{i}$, and it follows that $\chi_{i}\left(1+x^{i}\right)=-2^{i-1}$.

Finally, we compute $\chi_{i}\left(1+x^{j}\right)$ when $i>j$. If $\lambda=\lambda_{i}+\mu$ is a member of orbit $i$, then $\lambda\left(x^{j}\right)=\mu\left(x^{j}\right)$, and this is 0 for half of the possible functionals $\mu$, and it is equal to 1 for the remaining half. (This is because exactly half of the linear combinations of $\lambda_{k}$ with $k<i$ involve $\lambda_{j}$.) It follows that $\chi_{i}\left(1+x^{j}\right)=0$ in this case, and this completes the determination of the supercharacter table of $G=1+J$.

To illustrate, we give the table explicitly in the case $n=4$. The columns in the following array correspond to the five superclasses. The first entry in each column is the size of the superclass, and the second is a representative element. Of course, the character degrees, which are the sizes of the orbits on $J^{*}$, are the entries in the first column of the table.

TABLE 1

\begin{tabular}{|c||c|c|c|c|c|}
\hline & 1 & 1 & 2 & 4 & 8 \\
\hline & 1 & $1+x^{4}$ & $1+x^{3}$ & $1+x^{2}$ & $1+x$ \\
\hline \hline$\chi_{0}$ & 1 & 1 & 1 & 1 & 1 \\
\hline$\chi_{1}$ & 1 & 1 & 1 & 1 & -1 \\
\hline$\chi_{2}$ & 2 & 2 & 2 & -2 & 0 \\
\hline$\chi_{3}$ & 4 & 4 & -4 & 0 & 0 \\
\hline$\chi_{4}$ & 8 & -8 & 0 & 0 & 0 \\
\hline
\end{tabular}

Notice that in this family of examples, the sizes of the superclasses are equal to the degrees of the supercharacters, but this is not generally true, even when $G=1+J$ is abelian. Consider, for example, the nilpotent algebra $J$ with basis $\{x, y, z\}$, where products of basis vectors are zero except that $x y=z=y x$. Again working in the case $|F|=2$, it is easy to check that the (two-sided) $G$-orbits on $J$ are $\{0\},\{z\},\{x, x+z\},\{y, y+z\}$ and $\{x+y, x+y+z\}$, and thus $G$ has five superclasses, with sizes $1,1,2,2$ and 2 .

Now let $\{\lambda, \mu, \nu\}$ be the dual basis of $J^{*}$ corresponding to the given basis for $J$. It is not hard to check that the $G$-orbits on $J^{*}$ are $\{0\},\{\lambda\},\{\mu\},\{\lambda+\mu\}$ and $\{\nu, \nu+\lambda, \nu+\mu, \nu+\lambda+\mu\}$, and thus the degrees of the five supercharacters are $1,1,1,1$ and 4 . The full supercharacter table is presented below, where we have numbered the supercharacters (starting with $\chi_{0}$ ) in the order listed above for the 
corresponding orbits on $J^{*}$. As before, the first two entries in each column are the size and a representative element of the corresponding superclass.

TABLE 2

\begin{tabular}{|c||c|c|c|c|c|}
\hline & 1 & 1 & 2 & 2 & 2 \\
\hline & 1 & $1+z$ & $1+x$ & $1+y$ & $1+x+y$ \\
\hline \hline$\chi_{0}$ & 1 & 1 & 1 & 1 & 1 \\
\hline$\chi_{2}$ & 1 & 1 & -1 & 1 & -1 \\
\hline$\chi_{1}$ & 1 & 1 & 1 & -1 & -1 \\
\hline$\chi_{3}$ & 1 & 1 & -1 & -1 & 1 \\
\hline$\chi_{4}$ & 4 & -4 & 0 & 0 & 0 \\
\hline
\end{tabular}

Appendix D: Fourier analysis. For applications, the following reformulation of our results into the language of classical Fourier analysis is useful. Let $G$ be an arbitrary finite group, and let $M$ be the space of all complex valued functions on $G$. As we remarked in Section 5, there is a natural isomorphism $M \cong \mathbb{C} G$, where $\alpha \in M$ corresponds to $\sum_{g \in G} \alpha(g) g$ in the group algebra $\mathbb{C} G$. Multiplication in $\mathbb{C} G$ can be transferred to $M$ via this isomorphism, and the resulting operation on $M$ is convolution, defined by the formula

$$
(\alpha * \beta)(g)=\sum_{h \in G} \alpha\left(g h^{-1}\right) \beta(h) .
$$

Of course, the convolution $\alpha * \beta$ is linear in both variables.

Now suppose that $G$ admits a supercharacter theory, so that in particular we can apply all of the following to algebra groups. But note that by taking the "trivial" supercharacter theory, where the supercharacters are just the ordinary irreducible characters and the superclasses are the conjugacy classes, the following computations yield the "classical" formulas.

If $K$ and $L$ are superclasses of $G$ (in the sense of Section 2) let $\alpha$ and $\beta$ be their characteristic functions. Then $\alpha, \beta \in M$ correspond to $\widehat{K}$ and $\widehat{L}$ in $\mathbb{C} G$, and so $\alpha * \beta$ corresponds to $\widehat{K} \widehat{L} \in \mathbb{C} G$, which by Corollary 2.3 is a linear combination of superclass sums. It follows that $\alpha * \beta$ is a superclass function, and so by linearity, the subspace of $M$ consisting of superclass functions is closed under convolution. We know that the set $\mathcal{S}$ of supercharacters of $G$ is a basis for the set of superclass functions, so if we can compute the convolution of two supercharacters, the convolution operation on the full set of superclass functions will be determined.

Let $\chi$ be a supercharacter of $G$, so that $\chi$ is a multiple of a character of the form $\sigma_{X}$ for some subset $X \subseteq \operatorname{Irr}(G)$. In general, therefore, we can write $\chi=\left(1 / n_{\chi}\right) \sigma_{X}$ for some constant $n_{\chi}$, depending on $\chi$. (For algebra groups, we have $n_{\chi}=n_{\lambda}$ if $\chi=\chi_{\lambda}$, and in the situation of Section 2, we have $n_{\chi}=1$. Also, for the trivial supercharacter theory, where the supercharacters are just the irreducible characters, we have $n_{\chi}=\chi(1)$ for all $\chi \in \operatorname{Irr}(G)$.) Recall that $\left[\sigma_{X}, \sigma_{X}\right]=\sigma_{X}(1)$, and so $[\chi, \chi]=\sigma_{X}(1) / n^{2}=\chi(1) / n_{\chi}$.

Let $f_{X}$ be the idempotent corresponding to the supercharacter $\chi$, as in Section 2, and recall that

$$
f_{X}=\frac{1}{|G|} \sum_{g \in G} \overline{\sigma_{X}(g)}=\frac{n_{\chi}}{|G|} \sum_{g \in G} \overline{\chi(g)} g .
$$


The function $(n /|G|) \bar{\chi} \in M$, therefore, corresponds to the idempotent $f_{X} \in \mathbb{C} G$. Since the elements $f_{X} \in \mathbb{C} G$ are orthogonal idempotents, we deduce that

$$
\left(\frac{n_{\chi}}{|G|} \chi\right) *\left(\frac{n_{\psi}}{|G|} \psi\right)= \begin{cases}0 & \text { if } \psi \neq \chi \\ \left(n_{\chi} /|G|\right) \chi & \text { if } \psi=\chi\end{cases}
$$

for supercharacters $\chi$ and $\psi$. Thus

$$
\chi * \psi= \begin{cases}0 & \text { if } \psi \neq \chi \\ \left(|G| / n_{\chi}\right) \chi & \text { if } \psi=\chi .\end{cases}
$$

Now if $\alpha$ is an arbitrary superclass function on $G$, we can write $\alpha$ as a linear combination of the supercharacters $\chi \in \mathcal{S}$, and in fact, the coefficient of $\chi$ is exactly $[\alpha, \chi] /[\chi, \chi]=n_{\chi}[\alpha, \chi] / \chi(1)$. Since $\chi * \psi=0$ for distinct $\chi, \psi \in \mathcal{S}$, it follows that if $\alpha$ and $\beta$ are superclass functions, then

$$
\alpha * \beta=\sum_{\chi \in \mathcal{S}}\left(\frac{n_{\chi}}{\chi(1)}\right)^{2}[\alpha, \chi][\beta, \chi](\chi * \chi)=\sum_{\chi \in \mathcal{S}} \frac{|G| n_{\chi}}{\chi(1)^{2}}[\alpha, \chi][\beta, \chi] \chi .
$$

It follows from this that

$$
[(\alpha * \beta), \chi]=\frac{|G| n_{\chi}}{\chi(1)^{2}}[\alpha, \chi][\beta, \chi][\chi, \chi]=\frac{|G|}{\chi(1)}[\alpha, \chi][\beta, \chi] .
$$

Recall that we are writing $\mathcal{S}$ to denote the set of supercharacters of $G$. Given an arbitrary superclass function $\alpha$ on $G$, we define the Fourier transform $\widehat{\alpha}: \mathcal{S} \rightarrow \mathbb{C}$ by the formula

$$
\widehat{\alpha}(\chi)=\sum_{g \in G} \alpha(g) \bar{\chi}(g) .
$$

In other words, $\widehat{\alpha}(\chi)=|G|[\alpha, \chi]$. Also, since we can write

$$
\alpha=\sum_{\chi \in \mathcal{S}} \frac{[\alpha, \chi]}{[\chi, \chi]} \chi=\sum_{\chi \in \mathcal{S}} \frac{n_{\chi}[\alpha, \chi]}{\chi(1)} \chi
$$

we have

$$
\alpha=\frac{1}{|G|} \sum_{\chi \in \mathcal{S}} \frac{n_{\chi} \widehat{\alpha}(\chi)}{\chi(1)} \chi
$$

for all superclass functions $\alpha$. Furthermore, we see from our earlier calculation of $[(\alpha * \beta), \chi]$ that

$$
\widehat{\alpha * \beta}(\chi)=|G|[(\alpha * \beta), \chi]=\frac{1}{\chi(1)} \widehat{\alpha}(\chi) \widehat{\beta}(\chi),
$$

for superclass functions $\alpha$ and $\beta$.

Finally, consider a probability measure $Q$ on $G$, so that $\sum Q(g)=1$, where the sum runs over $g \in G$, and assume that $Q$ is a superclass function. We want to compute the "distance" from the $m$ th convolution power $Q^{* m}$ of $Q$ to the constant probability measure $u(g)=1 /|G|$.

First, recall from equation (1) that for $\chi \in \mathcal{S}$, we have $\chi * \chi=\left(G / n_{\chi}\right) \chi$, and thus

$$
\chi^{* m}=\left(\frac{|G|}{n_{\chi}}\right)^{m-1} \chi .
$$


It follows from equation (2) that if $\alpha$ is an arbitrary superclass function, then

$$
\alpha^{* m}=\sum_{\chi \in \mathcal{S}}\left(\frac{n_{\chi} \widehat{\alpha}(\chi)}{\chi(1)|G|}\right)^{m} \chi^{* m}=\frac{1}{|G|} \sum_{\chi \in \mathcal{S}} n_{\chi}\left(\frac{\widehat{\alpha}(\chi)}{\chi(1)}\right)^{m} \chi .
$$

We can now compute $\left\|Q^{* m}-u\right\|^{2}=\left[Q^{* m}-u, Q^{* m}-u\right]$ by applying (4) with $\alpha=Q$, and observing that $\widehat{Q}\left(1_{G}\right)=1$, where as usual, $1_{G}$ is the principal character of $G$. (Note that $1_{G} \in \mathcal{S}$ and that the associated constant $n_{1_{G}}=1$.) The coefficient of $1_{G}$ in the expansion of $Q^{* m}$ is $1 /|G|$, and since $u=(1 /|G|) 1_{G}$, we have

$$
Q^{* m}-u=\frac{1}{|G|} \sum_{\substack{\chi \in \mathcal{S} \\ \chi \neq 1_{G}}} n_{\chi}\left(\frac{\widehat{\alpha}(\chi)}{\chi(1)}\right)^{m} \chi
$$

and thus since $[\chi, \chi]=\chi(1) / n_{\chi}$, we have

$$
\left\|Q^{* m}-u\right\|^{2}=\frac{1}{|G|^{2}} \sum_{\substack{\chi \in \mathcal{S} \\ \chi \neq 1_{G}}} n_{\chi} \chi(1)\left(\frac{\widehat{Q}(\chi)}{\chi(1)}\right)^{2 m} .
$$

\section{REFERENCES}

[1] Carlos A. M. André, Basic characters of the unitriangular group, J. of Algebra 175 (1995), 287-319. MR1338979 (96h:20081a)

[2] Carlos A. M. André, Irreducible characters of finite algebra groups, Matrices and Group Representations Coimbra, 1998 Textos Mat. Sér B 19 (1999), 65-80. MR1773571 (2001g:20009)

[3] Carlos A. M. André, Basic characters of the unitriangular group (for arbitrary primes), Proc. Amer. Math Soc. 130 (2002), 1943-1954. MR1896026 (2003g:20075)

[4] E. Arias-Castro, Persi Diaconis and Richard Stanley, A super-class walk on upper-triangular matrices, J. of Algebra 278 (2004), 739-765. MR2071663 (2005f:60101)

[5] I. M. Isaacs, Character theory of finite groups, Dover, New York, 1994. MR1280461

[6] I. M. Isaacs, Characters of groups associated with finite algebras, J. of Algebra 177 (1995), 708-730. MR1358482 (96k:20011)

[7] I. M. Isaacs, Algebra groups, unpublished notes, 1997.

[8] I. M. Isaacs and Dikran Karagueuzian, Conjugacy in groups of upper triangular matrices, $J$. of Algebra 202 (1998), 704-711. MR1617655 (99b:20011)

[9] I. M. Isaacs and Dikran Karagueuzian, Erratum: Conjugacy in groups of upper triangular matrices, J. of Algebra 208 (1998), 722. MR1655475 (99g:20021)

[10] Andre Jaikin-Zapirain, A counterexample to the fake degree conjecture, Chebyshevskiı Sb. 5 (2004), 188-192. MR2098978 (2005g:20012)

[11] A. A. Kirillov, Variations on the triangular theme, Lie groups and Lie algebras: E. B. Dynkin's Seminar, 43-73, Amer. Math. Soc. Transl. Ser. 2, 169 Providence, RI, 1995. MR1364453 (97a:20072)

[12] I. G. Macdonald, Symmetric functions and Hall polynomials, Second edition. With contributions by A. Zelevinsky. Oxford Mathematical Monographs. Oxford University Press, New York, 1995. MR1354144 (96h:05207)

[13] Josu Sangroniz, Characters of algebra groups and unitriangular groups. Finite groups 2003, 335-349, Walter de Gruyter, Berlin, 2004. MR2125084 (2006e:20012)

[14] Ning Yan, Representation Theory of the finite unipotent linear groups, unpublished manuscript, 2001.

Department of Mathematics, Stanford University, 450 Serra Mall Bldg. 380, StanFORD, CALIFORNia 94305

E-mail address: diaconis@math.stanford.edu

Department of Mathematics, University of Wisconsin, 480 Lincoln Dr., Madison, WisCONSIN 53706

E-mail address: isaacs@math.wisc.edu 